\title{
A combined incremental-secant mean-field homogenization scheme with per-phase residual strains for elasto-plastic composites
}

\author{
L. Wu ${ }^{\mathrm{a}, *}$, L. Noels ${ }^{\mathrm{a}}$, L. Adam ${ }^{\mathrm{b}}$, I. Doghri ${ }^{\mathrm{b}, \mathrm{c}}$ \\ ${ }^{a}$ University of Liege, Department of Aeronautics and Mechanical Engineering - \\ Computational \& Multiscale Mechanics of Materials \\ Chemin des Chevreuils 1, B-4000 Liège, Belgium \\ ${ }^{b} e$-Xstream Engineering, Axis Park-Building $H$ \\ Rue Emile Francqui 9, B-1435 Mont-Saint-Guibert, Belgium \\ ${ }^{c}$ Université Catholique de Louvain, Bâtiment Euler, 1348 Louvain-la-Neuve, Belgium
}

\begin{abstract}
This paper presents an incremental secant mean-field homogenization (MFH) procedure for composites made of elasto-plastic constituents. In this formulation, the residual stress and strain states reached in the elasto-plastic phases upon a fictitious elastic unloading are considered as starting point to apply the secant method. The mean stress fields in the phases are then computed using secant tensors, which are naturally isotropic and enable to define the Linear-Comparison-Composite. The method, which remains simple in its formulation, is valid for general non-monotonic and non-proportional loading. It is applied on various problems involving elastic, elasto-plastic and perfectly-plastic phases, to demonstrate its accuracy compared to other existing MFH methods.
\end{abstract}

Keywords: Mean-Field Homogenization, Composites, Elasto-plasticity, Incremental-secant

\section{Introduction}

The direct numerical simulation of composite structures at fine scales being too expensive, emphasize was put during the last decades on the devel-

\footnotetext{
${ }^{*}$ Corresponding author, Phone: +32 436694 53, Fax: +32 43669505

Email address: L.Wu@ulg.ac.be, (L. Wu )
} 
opment of simplified homogenization methods. The latter are either (semi-) analytical or numerical and predict the macro or meso-scopic response of heterogeneous materials from their micro-structure and constituents properties at reduced computational cost while maintaining an acceptable degree of accuracy. Kanouté et al. (2009); Geers et al. (2010) presented an overview of the different homogenization methods. Among those methods, the mean-field homogenization (MFH) approach is an efficient semi-analytical framework for the modeling of multi-phase composites. MFH methods were first developed for linear elastic composite materials by extending the Eshelby (1957) single inclusion solution to multiple inclusions interacting in an average way in the composite. Most common extensions of the Eshelby solution are the MoriTanaka (M-T) scheme developed by Mori and Tanaka (1973) and Benveniste (1987) and the self-consistent scheme pioneered by Kröner (1958) and Hill (1965b).

MFH schemes were also extended to the non-linear range to account for non-linear behaviors, such as (visco-)plasticity or non-linear visco-elasticity, exhibited by the composite's constituents. Most of these extensions revolved around the definition of a so-called linear comparison composite (LCC) (Talbot and Willis, 1985, 1987; Ponte Castañeda, 1991, 1992; Talbot and Willis, 1992; Molinari et al., 2004), which is a virtual composite whose constituents linear behaviors match the linearized behavior of the real constituents for a given strain state. Such a LCC is used in the incremental formulation, proposed by Hill (1965a), which considers linearized relations between the stress and strain increments of the different constituents around their current strain states. Thus the former homogenization techniques for linear responses can still be used on the strain increments to predict the behaviors of highly nonlinear composites. Such an approach was applied to predict the meso-scale response of elasto-plastic composites by Pettermann et al. (1999); Doghri and Ouaar (2003); Doghri and Tinel (2005); Pierard and Doghri (2006b), in which case the behavior of the composite is written as $\Delta \bar{\sigma}=\bar{C}^{\text {tg }}: \Delta \bar{\varepsilon}$, where $\Delta \bar{\varepsilon}, \Delta \bar{\sigma}$ and $\bar{C}^{\text {tg }}$ are respectively macro-strain and stress increments, and a tangent operator. This incremental-tangent method can lead to too-stiff results unless some isotropic projections of the tangent operator are considered during the $\mathrm{M}-\mathrm{T}$ process, as shown by Doghri and Ouaar (2003); Pierard and Doghri (2006b). Another MFH approach is the so-called affine method, which was first proposed by Molinari et al. (1987, 2004) for visco-plastic materials, and which considers the total strain field instead of strain increments 
during the homogenization process. This approach was extended to elastoplastic materials by Zaoui and Masson (2002); Masson et al. (2000). For the affine method, the behavior of the composite is expressed as $\bar{\sigma}=\bar{C}: \bar{\varepsilon}+\tau$, where $\boldsymbol{\tau}$ is the polarization stress and where $\bar{C}$ can be different from the tangent moduli. Chaboche et al. (2005) showed that this method can lead to too stiff results when an anisotropic tangent operator is considered in the homogenization process. Different and often accurate affine methods for visco-plastic composites were proposed by Pierard and Doghri (2006a); Mercier and Molinari (2009); Doghri et al. (2010). The LCC can also be defined from a secant operator, as initially proposed by Berveiller and Zaoui (1978) for elasto-plastic materials. In this secant method the operator is the secant joining the origin to the current strain/stress state, and the response of the composite reads $\overline{\boldsymbol{\sigma}}=\overline{\boldsymbol{C}}^{\text {sec }}: \bar{\varepsilon}$, which limits the method to monotonic and proportional loading paths. Recently, Wu et al. (2012) -the authorshave proposed a non-local incremental-tangent $\mathrm{MFH}$ scheme accounting for damage. In that formulation, the incremental $\mathrm{MFH}$ approach is extended to account for the damage behavior happening in the matrix-material at the micro-scale. In order to avoid the strain/damage localization caused by the matrix material softening, a gradient-enhanced formulation (Peerlings et al., 2001; Engelen and Baaijens, 2003) was adopted during the homogenization process. In this formulation, the non-local accumulated plastic strain of the matrix is defined and depends on the local accumulated plastic strain and on its derivatives through the resolution of a new boundary value problem following the developments of Peerlings et al. (1996); Geers (1997); Peerlings et al. (1998).

Most MFH methods only consider first-moment-statistical values of the micro-strain and stress fields during the homogenization process. This can lead to poor predictions in the elasto-plastic case, as shown by Moulinec and Suquet (2003). This motivated to consider second-moment-statistical values (Ponte Castañeda, 1996) during the homogenization process. Such methods have been proposed for the secant formulations by Suquet (1995); Ponte Castañeda (2002a,b) and for the incremental-tangent formulation by Doghri et al. (2011). Suquet (1995) actually showed that the variational forms pioneered by Ponte Castañeda (1992) correspond to a second-order secant formulation, which was called modified secant. Recently, incremental variational formulations, which also correspond to a second-moment estimation, were proposed for visco-elastic composites by Lahellec and Suquet (2007a,b), for thermoelastic composites by Lahellec et al. (2011), for elasto- 
(visco-)plastic composites by Brassart et al. (2011, 2012), and for elastovisco-plastic composites with isotropic and kinematic hardening by Lahellec and Suquet (2013). The method proposed by Brassart et al. (2011, 2012) can also be seen as a secant method based on the elastic trial strain, instead of the total strain as in the original secant formulation, which allows the case of non-monotonic and non-radial loading conditions to be simulated.

Note that there exist other homogenization methods, which consider nonlinear effects, such as the transformation field analysis proposed by Dvorak (1992); Dvorak et al. (1994), which is a MFH scheme for elasto-plastic composites where a relaxation stress is defined due to the irreversible behavior in the matrix, leading to treat the interaction between the phases in a purely elastic way: $\overline{\boldsymbol{\sigma}}=\overline{\boldsymbol{C}}^{\mathrm{el}}:\left(\bar{\varepsilon}-\bar{\varepsilon}_{\text {res }}\right)$. Homogenization methods not based on mean-field were also developed such as the method of cells proposed by Lissenden and Arnold (1997); Aboudi et al. (2003), the unit cell finite element (FE)-based computations as performed by Wieckowski (2000); Segurado et al. (2002); Ji and Wang (2003); Carrere et al. (2004), or again the multiscale FE2 method pioneered by Kouznetsova et al. (2002, 2004) as a non-exhaustive list.

Although multiscale homogenization methods in general, and MFH schemes in particular, often exhibit an acceptable, sometimes even high, level of accuracy to capture the non-linear behavior of composites (Pierard et al., 2007b), some limitations, assumptions or complexity in the formulation remain in the existing methods. For example, as previously said, to remain accurate in the non-linear range, the incremental-tangent method requires the tangent-operator to be made isotropic during the M-T process, as discussed by Doghri and Ouaar (2003); Pierard and Doghri (2006b). Another limit of the incremental-tangent method appears when damage is considered. As explained by $\mathrm{Wu}$ et al. (2012), during the softening stage of the matrix, the fibers should see an elastic unloading due to the damaging process in the matrix, which cannot be modeled using the incremental approach. As a result, when compared to the direct numerical simulations of a representative volume element (RVE), the method remains accurate for low fiber ratios or low damage values only. In the variational approach proposed by Brassart et al. (2012), the plastic strain field at the beginning of each time interval is supposed to be uniform within the RVE, threatening the accuracy of the method in some cases, as when UD composites made of elastic fibers are loaded in the longitudinal direction. 
It is intended in this paper to propose what we believe is a new MFH approach for elasto-plastic materials. In this approach, at a given strain/stress state of the composite material, an unloading step is applied on the composite material level and the residual stresses are evaluated in both phases. The mean stress fields in the phases are computed using isotropic secant tensors, which can in turn be used to define the LCC. Contrarily to the incrementaltangent approach, there is no need to made isotropic the tensor used in the Eshelby term, as it is intrinsically so. In this formulation, the residual strains in the different phases, and thus the plastic residual strains, are piece-wise continuous, removing a major assumption in the variational approach proposed by Brassart et al. (2012). Another advantage of the method is expected when damage induced strain softening will be considered in a future work. Indeed, upon strain softening of the matrix, the fibers will be unloaded by the formulation, which should in turn improve the accuracy of the method for high volume fractions of fibers.

The paper is organized as follows. In Section 2, the key principles of MFH for non-linear behaviors are briefly recalled. Section 3 presents the proposed incremental-secant MFH for non-linear composites. In this formulation, the residual strain in a phase appears in the form of a residual stress, obtained after the unloading of the composite. It is shown in Section 4 that the method predicts accurate results compared to direct numerical simulations for a broad range of elastic and elasto-plastic composite materials.

\section{Generalities on MFH}

In this section, the prerequisites to the development of the new incrementalsecant multiscale $\mathrm{MFH}$ are summarized. In particular, the principle of the MFH method for two-phase composites is recalled.

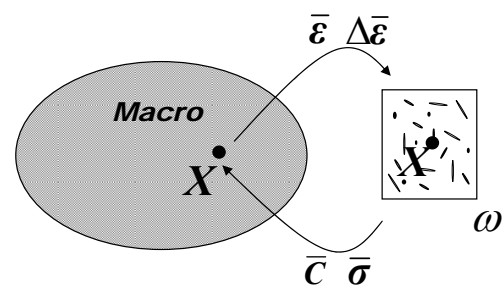

Figure 1: Multiscale method. 
In the multiscale approach illustrated in Fig. 1, at each macro-point $X$, the macro-strain $\bar{\varepsilon}$ is known, and the macro-stress $\bar{\sigma}$ is sought from a micro-scale boundary value problem (BVP), or vice-versa. At the microlevel, the macro-point is viewed as the center of a RVE of domain $x \in \omega$ and boundary $\partial \omega$. Considering adequate boundary conditions (BCs) on the RVE, the Hill-Mandell condition, expressing the equality between energies at both scales, transforms the relation between macro-strains $\bar{\varepsilon}$ and stresses $\bar{\sigma}$ into the relation between average strains $\langle\varepsilon\rangle$ and stresses $\langle\boldsymbol{\sigma}\rangle$ over the RVE, with $\langle f(\boldsymbol{x})\rangle=\frac{1}{V_{\omega}} \int_{\omega} f(\boldsymbol{x}) \mathrm{d} V$.

Considering a two-phase composite material with the phases volume ratios $v_{0}+v_{\mathrm{I}}=1$ (subscript 0 refers to the matrix and I to the inclusions), the macro-strains $\overline{\boldsymbol{\varepsilon}}$ and stresses $\overline{\boldsymbol{\sigma}}$ can be written in terms of the average values in the matrix subdomain $\omega_{0}$ and in the inclusions subdomain $\omega_{\mathrm{I}}$ as

$$
\begin{aligned}
& \bar{\varepsilon}=v_{0}\langle\varepsilon\rangle_{\omega_{0}}+v_{\mathrm{I}}\langle\varepsilon\rangle_{\omega_{\mathrm{I}}}, \quad \text { and } \\
& \overline{\boldsymbol{\sigma}}=v_{0}\langle\boldsymbol{\sigma}\rangle_{\omega_{0}}+v_{\mathrm{I}}\langle\boldsymbol{\sigma}\rangle_{\omega_{\mathrm{I}}},
\end{aligned}
$$

for both linear and non-linear frameworks.

For simplicity, in the following developments, the notations $\langle\bullet\rangle_{\omega_{i}}$ will be replaced by $\bullet_{i}$. Considering the so-called linear comparison composite, which is defined in the case of non-linear composites, the relation between the average incremental strains in the two phases depends on the chosen expressions of the virtual elastic operators $\overline{\boldsymbol{C}}_{0}^{\mathrm{LCC}}$ of the matrix phase and $\bar{C}_{\mathrm{I}}^{\mathrm{LCC}}$ of the inclusions phase I, leading to

$$
\Delta \varepsilon_{\mathrm{I}}=B^{\epsilon}\left(\mathrm{I}, \bar{C}_{0}^{\mathrm{LCC}}, \overline{\boldsymbol{C}}_{\mathrm{I}}^{\mathrm{LCC}}\right): \Delta \varepsilon_{0} .
$$

This equation describes the relation between the strain increment averages per phase through the strain concentration tensor $\boldsymbol{B}^{\epsilon}$, which expression depends on the assumptions made on the micro-mechanics. In this paper, we consider the Mori and Tanaka (1973) (M-T) model because it provides good predictions for two-phase composite materials for which the matrix can be clearly identified as discussed by Segurado and Llorca (2002). In this case, the strain concentration tensor reads

$$
\boldsymbol{B}^{\epsilon}=\left\{\boldsymbol{I}+\boldsymbol{S}:\left[\left(\overline{\boldsymbol{C}}_{0}^{\mathrm{LCC}}\right)^{-1}: \overline{\boldsymbol{C}}_{\mathrm{I}}^{\mathrm{LCC}}-\boldsymbol{I}\right]\right\}^{-1},
$$

where the Eshelby (1957) tensor $\boldsymbol{S}\left(\mathrm{I}, \overline{\boldsymbol{C}}_{0}^{\mathrm{LCC}}\right)$ depends on the geometry of the inclusions phase and on the virtual elastic operator $\overline{\boldsymbol{C}}_{0}^{\mathrm{LCC}}$. The expressions 
of the tensors $\overline{\boldsymbol{C}}_{0}^{\mathrm{LCC}}$ and $\overline{\boldsymbol{C}}_{\mathrm{I}}^{\mathrm{LCC}}$ depend on the chosen MFH process. In linear elasticity, these operators correspond to the elastic material moduli $C_{0}^{\mathrm{el}}$ and $\boldsymbol{C}_{\mathrm{I}}^{\mathrm{el}}$. In the classical incremental MFH method for non-linear materials, they correspond to the so-called comparison tangent operators $\overline{\boldsymbol{C}}_{0}^{\text {alg }}$ and $\overline{\boldsymbol{C}}_{\mathrm{I}}^{\text {alg }}$, which are uniform per phase, by design.

Note that when considering the incremental-tangent formulation, the Eshelby tensor $S\left(\mathrm{I}, \overline{\boldsymbol{C}}_{0}^{\text {iso }}\right)$ required to compute the Mori-Tanaka strain concentration tensor (4) is evaluated from an isotropic part $\overline{\boldsymbol{C}}_{0}^{\text {iso }}$ of $\overline{\boldsymbol{C}}_{0}^{\mathrm{LCC}}$, to improve prediction results. More details can be found in the work of Pierard and Doghri (2006b).

\section{New proposal: Incremental-secant MFH}

In this section, the new incremental-secant mean-field homogenization (MFH) scheme is developed for elasto-plastic composites. First, the secant formulation that will be considered to define the LCC is explained. Then, the MFH process is described in details.

\subsection{Incremental-secant moduli for rate-independent models}

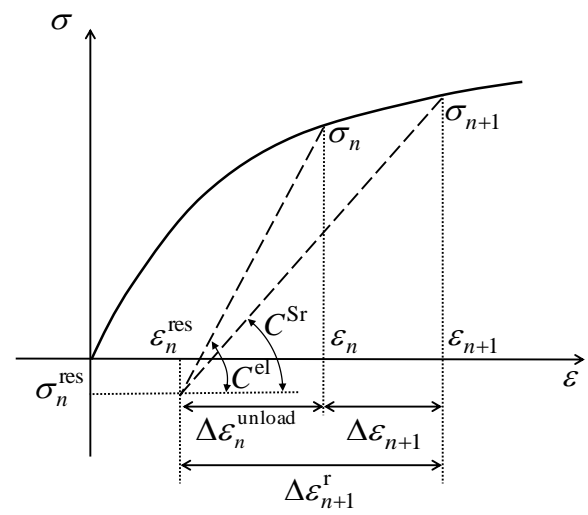

(a) Residual-secant

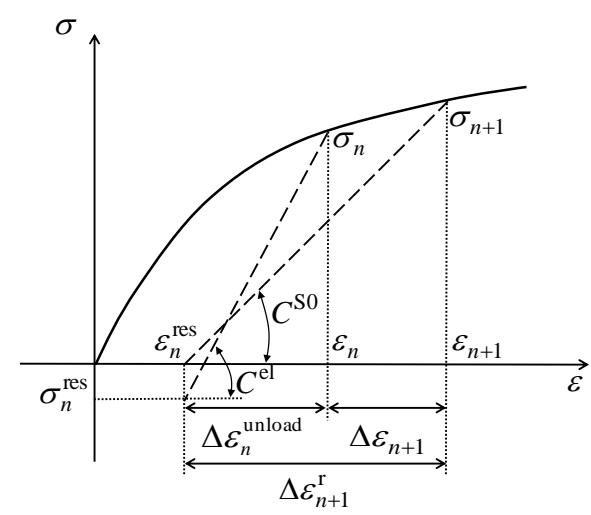

(b) Zero-secant

Figure 2: Definition of the incremental-secant formulation. (a) Definition of the residual strain and stress and of the residual secant operator. (b) Definition of the zero-secant operator.

The secant formulation that will be used in the MFH framework developed herein is introduced in this section. The equations derived herein can 
be related to the each phase of the composite. Considering a time interval $\left[t_{n}, t_{n+1}\right]$, with the total strain tensor $\varepsilon_{n}$ at time $t_{n}$ and the strain increment $\Delta \varepsilon_{n+1}$ resulting from the applied loading (for example from a finite element resolution) yields the strain tensor

$$
\varepsilon_{n+1}=\varepsilon_{n}+\Delta \varepsilon_{n+1},
$$

at time $t_{n+1}$, see Fig. 2(a). One can assume at time $t_{n}$ a residual strain tensor $\boldsymbol{\varepsilon}_{n}^{\text {res }}$ that corresponds to an elastic unloading from the stress state $\boldsymbol{\sigma}_{n}$ to a stress state $\boldsymbol{\sigma}_{n}^{\text {res }}$. Considering a heterogeneous material, this definition of the residual stress is related to each material phase but also to the homogenized material. During the homogenization process, the residual stress for the homogenized material will be null, but this will not be necessarily the case in the different phases. The same definitions also apply at time $t_{n+1}$.

The secant linearization of the elasto-plastic material is thus carried out in the time interval $\left[t_{n}, t_{n+1}\right]$ with the strain increment $\Delta \varepsilon_{n+1}^{\mathrm{r}}$, such that

$$
\varepsilon_{n+1}=\varepsilon_{n}^{\mathrm{res}}+\Delta \varepsilon_{n+1}^{\mathrm{r}} .
$$

The main idea developed in this paper is to define a LCC, subjected to a strain increment $\Delta \varepsilon_{n+1}^{\mathrm{r}}$, from which the stress tensor is computed. As illustrated in Fig. 2, two methods will be considered in this work: the residual-incremental-secant method, which evaluates the stress tensor from the residual stress arising upon virtual unloading, and the zero-incrementalsecant method, which evaluates the stress tensor from a zero-stress state. Both methods are now presented.

\subsubsection{Residual-incremental-secant approach}

Following Fig. 2(a), the new stress tensor could be defined from the stress increment $\Delta \boldsymbol{\sigma}_{n+1}^{\mathrm{r}}$, such that

$$
\boldsymbol{\sigma}_{n+1}=\boldsymbol{\sigma}_{n}^{\mathrm{res}}+\Delta \boldsymbol{\sigma}_{n+1}^{\mathrm{r}}
$$

where

$$
\Delta \sigma_{n+1}^{\mathrm{r}}=C^{\mathrm{Sr}}: \Delta \varepsilon_{n+1}^{\mathrm{r}} .
$$

In this last equation $C^{\mathrm{Sr}}$ is the residual-incremental-secant operator of the elasto-plastic material.

During the elastic regime, the elastic tensor $C^{\text {el }}$ can be used in the homogenization procedure. During elasto-plastic flow, the stress tensor $\boldsymbol{\sigma}_{n+1}$ is computed from the unloaded state in the following way: 


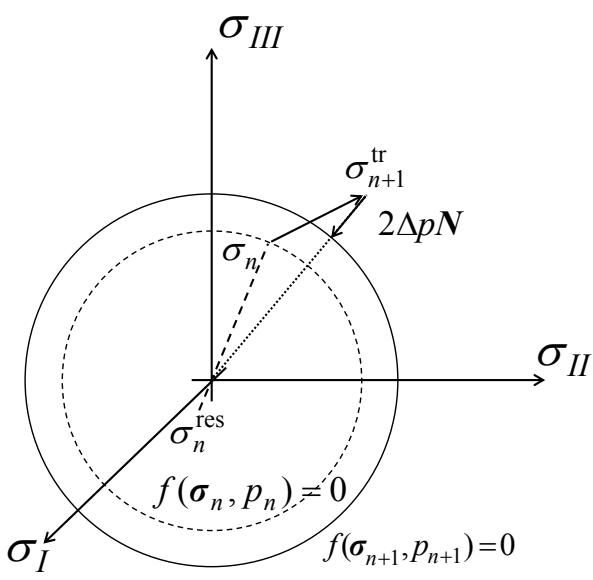

(a) Radial return mapping

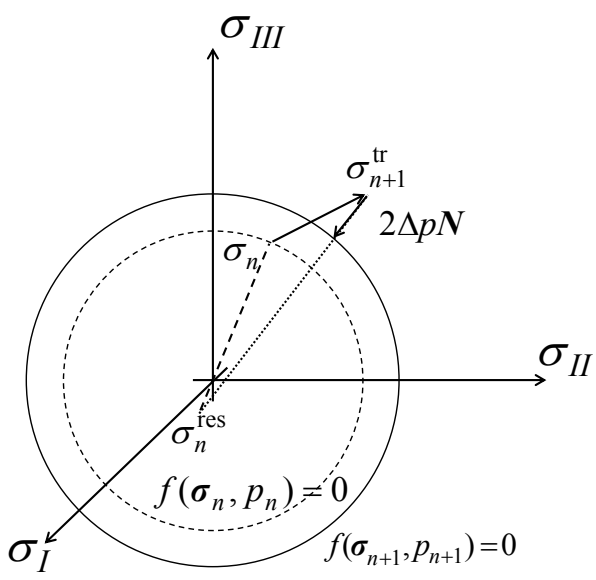

(b) Approximation

Figure 3: Plastic corrections in the stress space (a) Radial return mapping (b) Approximation (14).

- Evaluate the trial stress tensor from an elastic response:

$$
\begin{aligned}
\boldsymbol{\sigma}_{n+1}^{\mathrm{tr}} & =\sigma_{n}+C^{\mathrm{el}}: \Delta \varepsilon_{n+1}=\sigma_{n}+C^{\mathrm{el}}:\left(\Delta \varepsilon_{n+1}^{\mathrm{r}}+\varepsilon_{n}^{\mathrm{res}}-\varepsilon_{n}\right) \\
& =\sigma_{n}^{\mathrm{res}}+C^{\mathrm{el}}: \Delta \varepsilon_{n+1}^{\mathrm{r}} .
\end{aligned}
$$

- If the trial stress tensor respects the von Mises criterion

$$
f^{\operatorname{tr}}\left(\boldsymbol{\sigma}_{n+1}^{\mathrm{tr}}, p_{n}\right)=\left(\boldsymbol{\sigma}_{n+1}^{\mathrm{tr}}\right)^{\mathrm{eq}}-\sigma_{Y}-R\left(p_{n}\right) \leq 0,
$$

then the trial stress (9) is the solution and $\boldsymbol{C}^{\mathrm{Sr}}=\boldsymbol{C}^{\mathrm{el}}$. In Eq. (10), $\sigma_{Y}$ is the initial yield stress, $R(p)$ is the isotropic hardening law in terms of the accumulated plastic strain $p$, and the superscript "eq" refers to the equivalent von Mises effective stress.

- If the trial stress tensor does not respect the von Mises criterion, i.e. $f^{\text {tr }}>0$, then apply the plastic correction

$$
\sigma_{n+1}=\sigma_{n+1}^{\mathrm{tr}}-C^{\mathrm{el}}: \Delta \varepsilon^{\mathrm{p}}, \text { with } \Delta \varepsilon^{\mathrm{p}}=\Delta p \boldsymbol{N}_{n+1},
$$

where $N$ is the plastic flow direction. In this paper, we assume

$$
N_{n+1}=\frac{3}{2} \frac{\left(C^{\mathrm{Sr}}: \Delta \varepsilon_{n+1}^{\mathrm{r}}\right)^{\mathrm{dev}}}{\left(C^{\mathrm{Sr}}: \Delta \varepsilon_{n+1}^{\mathrm{r}}\right)^{\mathrm{eq}}}
$$


which satisfies $N: N=\frac{3}{2}$. Because of Eqs. (7-8), this last equation can be rewritten

$$
N_{n+1}=\frac{3}{2} \frac{\left(\boldsymbol{\sigma}_{n+1}-\boldsymbol{\sigma}_{n}^{\mathrm{res}}\right)^{\mathrm{dev}}}{\left(\boldsymbol{\sigma}_{n+1}-\boldsymbol{\sigma}_{n}^{\mathrm{res}}\right)^{\mathrm{eq}}} .
$$

When $\left(\boldsymbol{\sigma}_{n}^{\text {res }}\right)^{\text {dev }}=0$, this last equation corresponds to $\boldsymbol{N}_{n+1}=\left(\frac{\partial f(\boldsymbol{\sigma}, p)}{\partial \boldsymbol{\sigma}}\right)_{n+1}$ and we have the classical relation. When $\left(\boldsymbol{\sigma}_{n}^{\text {res }}\right)^{\text {dev }} \neq 0, \boldsymbol{N}_{n+1}$ is a first order approximation of the normal to the yield surface in the stress space, see Fig. 3. Indeed for infinitesimal strain increments $\Delta \varepsilon \rightarrow 0$, the reloading process $C^{\mathrm{el}}: \Delta \varepsilon_{n+1}^{\mathrm{r}}$ tends to the unloading increment $\boldsymbol{C}^{\mathrm{el}}: \Delta \boldsymbol{\varepsilon}^{\text {unload }}$, see Fig. 2(a), and thus $\boldsymbol{N}_{n+1}$ tends to the normal to the yield surface.

At the trial state, using Eq. (9) allows Eq. (12) to be rewritten as

$$
N_{n+1}^{\mathrm{tr}}=\frac{3}{2} \frac{\left(C^{\mathrm{el}}: \Delta \varepsilon_{n+1}^{\mathrm{r}}\right)^{\mathrm{dev}}}{\left(\boldsymbol{C}^{\mathrm{el}}: \Delta \varepsilon_{n+1}^{\mathrm{r}}\right)^{\mathrm{eq}}}=\frac{3}{2} \frac{\left(\boldsymbol{\sigma}_{n+1}^{\mathrm{tr}}-\boldsymbol{\sigma}_{n}^{\mathrm{res}}\right)^{\mathrm{dev}}}{\left(\boldsymbol{\sigma}_{n+1}^{\mathrm{tr}}-\boldsymbol{\sigma}_{n}^{\mathrm{res}}\right)^{\mathrm{eq}}} .
$$

Note that since $\boldsymbol{C}^{\mathrm{el}}$ is isotropic we have $\boldsymbol{C}^{\mathrm{el}}:\left(\Delta p \boldsymbol{N}_{n+1}\right)=2 \mu^{\mathrm{el}} \boldsymbol{N}_{n+1} \Delta p$ and Eq. (11) becomes

$$
\left(\boldsymbol{\sigma}_{n+1}\right)^{\mathrm{dev}}=\left(\boldsymbol{\sigma}_{n+1}^{\mathrm{tr}}\right)^{\mathrm{dev}}-2 \mu^{\mathrm{el}} \boldsymbol{N}_{n+1} \Delta p
$$

which can be rewritten as

$$
\left(\boldsymbol{\sigma}_{n+1}-\boldsymbol{\sigma}_{n}^{\mathrm{res}}\right)^{\mathrm{dev}}=\left(\boldsymbol{\sigma}_{n+1}^{\mathrm{tr}}-\boldsymbol{\sigma}_{n}^{\mathrm{res}}\right)^{\mathrm{dev}}-2 \mu^{\mathrm{el}} \boldsymbol{N}_{n+1} \Delta p,
$$

or, using the definitions (13) and (14), as

$$
\underbrace{\left[\left(\boldsymbol{\sigma}_{n+1}-\boldsymbol{\sigma}_{n}^{\mathrm{res}}\right)^{\mathrm{eq}}+3 \mu^{\mathrm{el}} \Delta p\right]}_{\geq 0} \boldsymbol{N}_{n+1}=\underbrace{\left(\boldsymbol{\sigma}_{n+1}^{\mathrm{tr}}-\boldsymbol{\sigma}_{n}^{\mathrm{res}}\right)^{\mathrm{eq}}}_{\geq 0} \boldsymbol{N}_{n+1}^{\mathrm{tr}} \text {. }
$$

Since Eqs. (12) and (14) imply $\boldsymbol{N}_{n+1}: \boldsymbol{N}_{n+1}=\frac{3}{2}=\boldsymbol{N}_{n+1}^{\mathrm{tr}}: \boldsymbol{N}_{n+1}^{\mathrm{tr}}$, then Eq. (17) results in

$$
\begin{aligned}
& \left(\boldsymbol{\sigma}_{n+1}-\boldsymbol{\sigma}_{n}^{\mathrm{res}}\right)^{\mathrm{eq}}+3 \mu^{\mathrm{el}} \Delta p=\left(\boldsymbol{\sigma}_{n+1}^{\mathrm{tr}}-\boldsymbol{\sigma}_{n}^{\mathrm{res}}\right)^{\mathrm{eq}} \text { and } \\
& \boldsymbol{N}_{n+1}=\boldsymbol{N}_{n+1}^{\mathrm{tr}} .
\end{aligned}
$$

The elasto-plastic scheme consists of solving the equations (18) and $f\left(\boldsymbol{\sigma}_{n+1}, p_{n+1}\right)=0$, with $p_{n+1}=p_{n}+\Delta p$, in terms of $\Delta p$ and $\boldsymbol{\sigma}_{n+1}^{\text {eq }}$. 
- Knowing $\boldsymbol{\sigma}_{n+1}$, compute the residual-incremental-secant operator of the linear comparison material as follows. From Eqs. (7) and (11) one has

$$
\Delta \boldsymbol{\sigma}_{n+1}^{\mathrm{r}}=\boldsymbol{C}^{\mathrm{Sr}}: \Delta \varepsilon_{n+1}^{\mathrm{r}}=\boldsymbol{C}^{\mathrm{el}}: \Delta \varepsilon_{n+1}^{\mathrm{r}}-2 \mu^{\mathrm{el}} \Delta p \boldsymbol{N}_{n+1},
$$

which becomes after using (14) and (19),

$$
\Delta \boldsymbol{\sigma}_{n+1}^{\mathrm{r}}=\left[\boldsymbol{C}^{\mathrm{el}}-3 \mu^{\mathrm{el}} \Delta p \frac{\boldsymbol{I}^{\mathrm{dev}}: \boldsymbol{C}^{\mathrm{el}}}{\left(\boldsymbol{C}^{\mathrm{el}}: \Delta \varepsilon_{n+1}^{\mathrm{r}}\right)^{\mathrm{eq}}}\right]: \Delta \varepsilon_{n+1}^{\mathrm{r}}=C^{\mathrm{Sr}}: \Delta \varepsilon_{n+1}^{\mathrm{r}} .
$$

For J2-elasto-plastic material, since $C^{\text {el }}$ is isotropic, the residual-incremental-secant operator of the linear comparison material $C^{\mathrm{Sr}}$ is also isotropic. Moreover, as $\boldsymbol{C}^{\mathrm{el}}=3 \kappa^{\mathrm{el}} \boldsymbol{I}^{\mathrm{vol}}+2 \mu^{\mathrm{el}} \boldsymbol{I}^{\mathrm{dev}}$, one can directly deduce

$$
\boldsymbol{C}^{\mathrm{Sr}}=3 \kappa^{\mathrm{r}} \boldsymbol{I}^{\mathrm{vol}}+2 \mu_{s}^{\mathrm{r}} \boldsymbol{I}^{\mathrm{dev}}
$$

with

$$
\begin{aligned}
\kappa^{\mathrm{r}} & =\kappa^{\mathrm{el}}, \text { and } \\
\mu_{s}^{\mathrm{r}} & =\mu^{\mathrm{el}}-\frac{3 \mu^{\mathrm{el}^{2}} \Delta p}{\left(\boldsymbol{C}^{\mathrm{el}}: \Delta \boldsymbol{\varepsilon}_{n+1}^{\mathrm{r}}\right)^{\mathrm{eq}}}=\mu^{\mathrm{el}}-\frac{3 \mu^{\mathrm{el}^{2}} \Delta p}{\left(\boldsymbol{\sigma}_{n+1}-\boldsymbol{\sigma}_{n}^{\mathrm{res}}\right)^{\mathrm{eq}}} .
\end{aligned}
$$

Note that on the one hand since $\left(C^{\mathrm{el}}: \Delta \varepsilon_{n+1}^{\mathrm{r}}\right)^{\mathrm{eq}}=3 \mu^{\mathrm{el}}\left(\Delta \varepsilon_{n+1}^{\mathrm{r}}\right)^{\mathrm{eq}}$, this last result can be rewritten $\mu_{s}^{\mathrm{r}}=\mu^{\mathrm{el}}\left(1-\frac{\Delta p}{\left(\Delta \varepsilon_{n+1}^{\mathrm{r}}\right)^{\mathrm{eq}}}\right)$. On the other hand, in the variational incremental MFH proposed by Brassart et al. (2011), the shear modulus in one phase used to define the LCC was found to be $\mu_{s}^{\mathrm{r}}=\mu^{\mathrm{el}}\left(1-\frac{\Delta p}{\sqrt{\left\langle\left(\boldsymbol{\varepsilon}_{n+1}-\boldsymbol{\varepsilon}_{n}^{\mathrm{p}}\right)^{\text {eq2}}\right\rangle_{\omega_{r}}}}\right)$. Besides this secondmoment form, it appears that both formulations have similarities. Indeed in case of unloading such that $\boldsymbol{\sigma}_{n}^{\text {res }}=0$ in the incremental-secant approach, one has $\Delta \varepsilon_{n+1}^{\mathrm{r}}=\varepsilon_{n+1}-\varepsilon_{n}^{\mathrm{p}}$.

- Practically the shear moduli of the virtual elastic material can be obtained by decomposing the increments of the stress and strain tensors into the hydrostatic and deviatoric parts:

$$
\Delta \boldsymbol{\sigma}^{\mathrm{r}}=\Delta \sigma^{m} \mathbf{1}+\Delta s \quad \text { and } \quad \Delta \varepsilon^{\mathrm{r}}=\Delta \varepsilon^{m} \mathbf{1}+\Delta \boldsymbol{e},
$$


where $\Delta \sigma^{m}=\frac{1}{3} \operatorname{tr}\left(\Delta \boldsymbol{\sigma}^{\mathrm{r}}\right), \Delta \boldsymbol{s}=\Delta \boldsymbol{\sigma}^{\mathrm{r}}-\Delta \sigma^{m} \mathbf{1}, \Delta \varepsilon^{m}=\frac{1}{3} \operatorname{tr}\left(\Delta \boldsymbol{\varepsilon}^{\mathrm{r}}\right)$, and where $\Delta \boldsymbol{e}=\Delta \varepsilon^{\mathrm{r}}-\Delta \varepsilon^{m} \mathbf{1}$, see Appendix $\mathrm{A}$ for the notations. Indeed, the increments of the von Mises equivalent stress and strain are respectively given by

$$
\Delta \sigma^{\mathrm{eq}}=\left(\frac{3}{2} \Delta s: \Delta s\right)^{1 / 2} \quad \text { and } \quad \Delta \varepsilon^{\mathrm{eq}}=\left(\frac{2}{3} \Delta \boldsymbol{e}: \Delta \boldsymbol{e}\right)^{1 / 2}
$$

and one has directly ${ }^{1}$

$$
3 \mu_{s}^{\mathrm{r}}=\frac{\Delta \sigma^{\mathrm{eq}}}{\Delta \varepsilon^{\mathrm{eq}}}
$$

- Evaluate the derivation of the operator (22) following Appendix B.1.

\subsubsection{Zero-incremental-secant approach}

Anticipating on the development of the new incremental-secant $\mathrm{MFH}$, and as it will be shown in Section 4.1, when defining the LCC, it can be advantageous to modify the residual-incremental-secant approach, following the suggestion in Fig. 2(b). The approach follows closely Section 3.1.1, but with the omission of the residual stress, in which case the plastic flow direction (13) is rigorously normal to the yield surface.

\subsubsection{Summary of the incremental-secant formulations}

The stress tensor of an elasto-plastic phase at time $t_{n+1}$ is formulated using the incremental secant operator of the isotropic-linear comparison material as

$\boldsymbol{\sigma}_{n+1}= \begin{cases}\boldsymbol{\sigma}_{n}^{\mathrm{res}}+C^{\mathrm{Sr}}: \Delta \varepsilon_{n+1}^{\mathrm{r}} & \text { for the residual-incremental-secant method; } \\ C^{\mathrm{S} 0}: \Delta \varepsilon_{n+1}^{\mathrm{r}} & \text { for the zero-incremental-secant method }\end{cases}$

In the forthcoming Section 4.1, a more detailed discussion illustrated by numerical examples will be given to justify the choice of the incrementalsecant operators to be considered in the matrix and in the inclusions phases.

\subsection{MFH scheme}

In this section, the new incremental-secant $\mathrm{MFH}$ is developed. Toward this end, the secant forms (28) will be considered to define the LCC. Unless

\footnotetext{
${ }^{1}$ If $\Delta \varepsilon^{\mathrm{eq}}=0$, the indefiniteness is solved by considering $\mu_{s}^{\mathrm{r}}=\mu^{\mathrm{el}}$
} 
the expressions need to be particularized to the residual-incremental-secant or to the zero-incremental-secant forms, the isotropic linear comparison stiffness

$$
\boldsymbol{C}^{\mathrm{S}}=3 \kappa \boldsymbol{I}^{\mathrm{vol}}+2 \mu_{s} \boldsymbol{I}^{\mathrm{dev}}
$$

will substitute to either $C^{\mathrm{Sr}}$ or $\boldsymbol{C}^{\mathrm{S} 0}$. Similarly, $\mu_{s}$ holds for either $\mu_{s}^{\mathrm{r}}$ or for $\mu_{s}^{0}$, while $\kappa=\kappa^{0}=\kappa^{\mathrm{r}}=\kappa^{\mathrm{el}}$, where superscript 0 refers to the values of the zeroincremental-secant approach. During the $\mathrm{MFH}$ the comparison operators are constructed as uniform by design on each phase $r$ and are thus denoted $\overline{\boldsymbol{C}}_{r}^{\mathrm{S}}$.

Considering a time interval $\left[t_{n}, t_{n+1}\right]$, the known data are the macro-total strain tensor at time $t_{n}, \bar{\varepsilon}_{n}$, the strain increment $\Delta \bar{\varepsilon}_{n+1}$, and the internal variables at time $t_{n}$. The latter include the internal variables, $\eta_{\mathrm{I}_{n}}$, of the inclusions and, $\eta_{0_{n}}$, of the matrix constitutive models, but also the residual variables computed from the elastic unloading step (i.e. $\overline{\boldsymbol{\sigma}}_{n}^{\text {res }}=0$ ): the residual strains in the composite, $\bar{\varepsilon}_{n}^{\text {res }}$, in the inclusions phase, $\varepsilon_{\mathrm{I}_{n}}^{\text {res }}$, in the matrix phase, $\boldsymbol{\varepsilon}_{0}{ }_{n}^{\text {res }}$, and the residual stresses in the inclusions phase, $\boldsymbol{\sigma}_{\mathrm{I}_{n}}^{\text {res }}$, and in the matrix phase, $\boldsymbol{\sigma}_{0_{n}}^{\text {res }}$.

It needs to be clarified that, in the developed incremental-secant method, the strain increment $\Delta \bar{\varepsilon}_{n+1}$ applied to the RVE is obtained from the FE resolution, while the strain increment $\Delta \bar{\varepsilon}_{n+1}^{\mathrm{r}}$ is used to define the LCC in the $\mathrm{MFH}$ procedure. This is an assumption as one cannot prove the existence of a displacement field increment compatible with $\Delta \bar{\varepsilon}_{n+1}^{\mathrm{r}}$.

Combining (5) and (6) for the homogenized material, one has

$$
\Delta \bar{\varepsilon}_{n+1}^{\mathrm{r}}=\bar{\varepsilon}_{n}+\Delta \bar{\varepsilon}_{n+1}-\bar{\varepsilon}_{n}^{\mathrm{res}} .
$$

The explicit evaluation of $\bar{\varepsilon}_{n}^{\text {res }}$ is described in the details of the MFH process reported here below.

The MFH process is stated by Eqs. (1) - (4). In this formalism, each phase $r$ will see a strain increment $\Delta \overline{\boldsymbol{\varepsilon}}_{r n+1}^{\mathrm{r}}$ to be applied from the elasticallyunloaded state, and from which a stress state

$$
\boldsymbol{\sigma}_{r n+1}=\mathcal{F}_{r}\left(\Delta \varepsilon_{r n+1}^{\mathrm{r}} ; \eta_{r_{n}}, \boldsymbol{\varepsilon}_{r_{n}}^{\mathrm{res}}, \boldsymbol{\sigma}_{r_{n}}^{\mathrm{res}}\right)
$$

and an incremental-secant tensor

$$
C_{r}^{\mathrm{S}}=\mathcal{G}_{r}\left(\Delta \varepsilon_{r n+1}^{\mathrm{r}} ; \eta_{r_{n}}, \varepsilon_{r_{n}}^{\mathrm{res}}, \boldsymbol{\sigma}_{r_{n}}^{\mathrm{res}}\right)
$$


which follows Eq. (28), can be obtained from the constitutive model provided in Section 3.1. The system of equations can thus be rewritten as

$$
\begin{aligned}
\Delta \bar{\varepsilon}_{n+1}^{\mathrm{r}} & =v_{0} \Delta \varepsilon_{0 n+1}^{\mathrm{r}}+v_{\mathrm{I}} \Delta \varepsilon_{\mathrm{I} n+1}^{\mathrm{r}}, \\
\overline{\boldsymbol{\sigma}}_{n+1} & =v_{0} \boldsymbol{\sigma}_{0 n+1}+v_{\mathrm{I}} \boldsymbol{\sigma}_{\mathrm{I} n+1},
\end{aligned}
$$

with the relation between the strain increments reading

$$
\Delta \varepsilon_{\mathrm{I} n+1}^{\mathrm{r}}=B^{\epsilon}\left(\mathrm{I}, \bar{C}_{0}^{\mathrm{S}}, \bar{C}_{\mathrm{I}}^{\mathrm{S}}\right): \Delta \varepsilon_{0 n+1}^{\mathrm{r}} .
$$

To complete these equations, the unloaded state is defined by

$$
\overline{\boldsymbol{\sigma}}^{\mathrm{res}}=v_{0} \boldsymbol{\sigma}_{0}^{\mathrm{res}}+v_{\mathrm{I}} \boldsymbol{\sigma}_{\mathrm{I} n}^{\mathrm{res}}=0 .
$$

To reach this virtual unloaded state, the composite material and each phase are assumed to obey an elastic behavior. As the unloaded state is a virtual state used to define the LCC, this assumption can always be made. This elastic unloading is thus performed from the state at time $t_{n}$ by solving the following system of equations:

$$
\begin{aligned}
& \underbrace{\bar{\varepsilon}_{n}-\bar{\varepsilon}_{n}^{\text {res }}}_{\Delta \bar{\varepsilon}_{n}^{\text {uload }}}=v_{0} \underbrace{\left(\varepsilon_{0 n}-\varepsilon_{0}^{\text {res }}\right)}_{\Delta \varepsilon_{0}^{\text {unload }}{ }_{n}}+v_{\mathrm{I}} \underbrace{\left(\varepsilon_{\mathrm{I} n}-\varepsilon_{\mathrm{I}}^{\text {res }} n\right)}_{\Delta \varepsilon_{\mathrm{I}}^{\text {unload }}{ }_{n}}, \\
& 0=\overline{\boldsymbol{\sigma}}_{n}^{\mathrm{res}}=v_{0}\left(\boldsymbol{\sigma}_{0 n}-\boldsymbol{C}_{0}^{\mathrm{el}}: \Delta \varepsilon_{0}^{\mathrm{unload}}{ }_{n}\right)+v_{\mathrm{I}}\left(\boldsymbol{\sigma}_{\mathrm{I} n}-\boldsymbol{C}_{\mathrm{I}}^{\mathrm{el}}: \Delta \varepsilon_{\mathrm{I}}^{\mathrm{unload}}{ }_{n}\right),
\end{aligned}
$$

with the relation between the unloading strain increments reading

$$
\Delta \varepsilon_{\mathrm{I}}^{\text {unload }}{ }_{n}=\boldsymbol{B}^{\epsilon}\left(\mathrm{I}, \boldsymbol{C}_{0}^{\mathrm{el}}, \boldsymbol{C}_{\mathrm{I}}^{\mathrm{el}}\right): \Delta \varepsilon_{0}^{\mathrm{unload}}{ }_{n} .
$$

In this paper, a "First-order moment" method is considered, and the $\mathrm{MFH}$ process is described as follows.

- Initialize the strain increment in inclusions from the composite strain increment $\Delta \bar{\varepsilon}_{n+1}^{\mathrm{r}}(30): \Delta \bar{\varepsilon}_{n+1}^{\mathrm{r}} \rightarrow \Delta \varepsilon_{\mathrm{I}_{n+1}}^{\mathrm{r}}$.

- Follow the iterations process (upper indices $(i)$ for values at iteration $i$ of time $t_{n+1}$ are omitted for simplicity):

1. Call the constitutive material functions (31-32) of the real inclusions material with, as input, the strain tensor increment in the inclusions phase $\Delta \varepsilon_{\mathrm{I} n+1}^{\mathrm{r}}$ and the internal variables $\eta_{\mathrm{I}_{n}}, \varepsilon_{\mathrm{I}_{n}}^{\text {res }}, \boldsymbol{\sigma}_{\mathrm{I}_{n}}^{\text {res }}$ at time $t_{n}$. The output is the updated stress $\sigma_{\mathrm{I} n+1}$, the internal variables at time $t_{n+1}$, and the incremental-secant operator $\overline{\boldsymbol{C}}_{\mathrm{I} n+1}^{\mathrm{S}}$ for the inclusions phase. In case there is no plastic flow, we use $\bar{C}_{\mathrm{I} n+1}^{\mathrm{S}}=C_{\mathrm{I}}^{\mathrm{el}}$, which is always the case for elastic materials. 
2. Compute the average strain in the matrix phase:

$$
\Delta \varepsilon_{0 n+1}^{\mathrm{r}}=\left(\Delta \bar{\varepsilon}_{n+1}^{\mathrm{r}}-v_{\mathrm{I}} \Delta \varepsilon_{\mathrm{I} n+1}^{\mathrm{r}}\right) / v_{0}
$$

3. Call the constitutive material functions (31-32) of the real matrix material with, as input, the strain tensor increment in the matrix phase $\Delta \varepsilon_{0 n+1}^{\mathrm{r}}$ and the internal variables $\eta_{0_{n}}, \varepsilon_{0_{n}}^{\text {res }}, \boldsymbol{\sigma}_{0_{n}}^{\text {res }}$ at time $t_{n}$. The output is the updated stress $\boldsymbol{\sigma}_{0 n+1}$, the internal variables at time $t_{n+1}$, and the incremental-secant operator $\overline{\boldsymbol{C}}_{0 n+1}^{\mathrm{S}}$ for the matrix phase. In case there is no plastic flow, we use $\bar{C}_{0 n+1}^{\mathrm{S}}=C_{0}^{\mathrm{el}}$.

4. Predict the Eshelby tensor $S\left(\mathrm{I}, \bar{C}_{0 n+1}^{\mathrm{S}}\right)$ using the secant operator of the matrix phase.

5. Applying a similar technique to the one detailed by $\mathrm{Wu}$ et al. (2012), Eq. (35) corresponds to satisfying $\boldsymbol{F}=0$, where $\boldsymbol{F}$ is the stress residual vector. For a time interval $\left[t_{n}, t_{n+1}\right]$, where $\Delta \bar{\varepsilon}_{n+1}^{\mathrm{r}}$ is constant, compute the stress residual vector in inclusions as, see Appendix C for details,

$$
\begin{aligned}
\boldsymbol{F}= & \overline{\boldsymbol{C}}_{0 n+1}^{\mathrm{S}}:\left[\Delta \varepsilon_{\mathrm{I}_{n+1}}^{\mathrm{r}}-\frac{1}{v_{0}} S^{-1}:\left(\Delta \varepsilon_{\mathrm{I}_{n+1}}^{\mathrm{r}}-\Delta \bar{\varepsilon}_{n+1}^{\mathrm{r}}\right)\right] \\
& -\overline{\boldsymbol{C}}_{\mathrm{I} n+1}^{\mathrm{S}}: \Delta \varepsilon_{\mathrm{I} n+1}^{\mathrm{r}}
\end{aligned}
$$

6. Check if the residual $|\boldsymbol{F}| \leq$ Tol. If so exit the loop.

7. Else, compute the Jacobian $\boldsymbol{J}$ matrix at constant $\Delta \bar{\varepsilon}_{n+1}^{\mathrm{r}}$, such that $\mathrm{d} \boldsymbol{F}=\boldsymbol{J}: \mathrm{d} \boldsymbol{\varepsilon}_{\mathrm{I}}^{\mathrm{r} 2}$, following the details given in Appendix C.

8. Correct the strain increment in inclusions

$$
\Delta \varepsilon_{\mathrm{I} n+1}^{\mathrm{r}} \leftarrow \Delta \varepsilon_{\mathrm{I} n+1}^{\mathrm{r}}+\boldsymbol{c}_{\varepsilon_{\mathrm{I}}} \text { with } \boldsymbol{c}_{\varepsilon_{\mathrm{I}}}=-\boldsymbol{J}^{-1}: \boldsymbol{F}
$$

then start a new iteration (go to step 1).

- After convergence, compute ${ }^{3}$

\footnotetext{
${ }^{2}$ Note that the derivative with respect to $\Delta \varepsilon_{r}^{\mathrm{r}}$ has the same expression as the derivative with respect to $\varepsilon_{r}$

${ }^{3}$ In this formalism, we do not need to evaluate explicitly the strain concentration tensor, nor the macro-moduli, but they follow directly from $\boldsymbol{B}^{\epsilon}=\left\{\boldsymbol{I}+\boldsymbol{S}:\left[\left(\overline{\boldsymbol{C}}_{0 n+1}^{\mathrm{S}}\right)^{-1}: \overline{\boldsymbol{C}}_{\mathrm{I} n+1}^{\mathrm{S}}-\right.\right.$ $\boldsymbol{I}]\}^{-1}$, and $\overline{\boldsymbol{C}}_{n+1}=\left[v_{\mathrm{I}} \overline{\boldsymbol{C}}_{\mathrm{I} n+1}^{\mathrm{S}}: \boldsymbol{B}^{\epsilon}+v_{0} \overline{\boldsymbol{C}}_{0 n+1}^{\mathrm{S}}\right]:\left[v_{1} \boldsymbol{B}^{\epsilon}+v_{0} \boldsymbol{I}\right]^{-1}$, respectively.
} 
1. The homogenized stress

$$
\overline{\boldsymbol{\sigma}}_{n+1}=v_{0} \boldsymbol{\sigma}_{0 n+1}+v_{\mathrm{I}} \boldsymbol{\sigma}_{\mathrm{I} n+1},
$$

and the internal variables.

2. The "consistent" ${ }^{4}$ linearization of the homogenized stress from (6) and (28)

$$
\begin{aligned}
\delta \overline{\boldsymbol{\sigma}}= & v_{\mathrm{I}} \delta \boldsymbol{\sigma}_{\mathrm{I}}+v_{0} \delta \boldsymbol{\sigma}_{0} \\
= & v_{\mathrm{I}} \frac{\partial\left(\overline{\boldsymbol{C}}_{\mathrm{I}}^{\mathrm{S}}: \Delta \varepsilon_{\mathrm{I}}^{\mathrm{r}}\right)}{\partial \boldsymbol{\varepsilon}_{\mathrm{I}}}: \delta \varepsilon_{\mathrm{I}}+v_{0} \frac{\partial\left(\overline{\boldsymbol{C}}_{0}^{\mathrm{S}}: \Delta \varepsilon_{0}^{\mathrm{r}}\right)}{\partial \boldsymbol{\varepsilon}_{0}}: \delta \varepsilon_{0} \\
= & {\left[v_{\mathrm{I}}\left(\overline{\boldsymbol{C}}_{\mathrm{I}}^{\mathrm{S}}+\frac{\partial \overline{\boldsymbol{C}}_{\mathrm{I}}^{\mathrm{S}}}{\partial \varepsilon_{\mathrm{I}}}: \Delta \varepsilon_{\mathrm{I}}^{\mathrm{r}}\right): \frac{\partial \varepsilon_{\mathrm{I}}}{\partial \overline{\boldsymbol{\varepsilon}}}+\right.} \\
& \left.v_{0}\left(\overline{\boldsymbol{C}}_{0}^{\mathrm{S}}+\frac{\partial \overline{\boldsymbol{C}}_{0}^{\mathrm{S}}}{\partial \varepsilon_{0}}: \Delta \varepsilon_{0}^{\mathrm{r}}\right): \frac{\partial \boldsymbol{\varepsilon}_{0}}{\partial \bar{\varepsilon}}\right]: \delta \overline{\boldsymbol{\varepsilon}} .
\end{aligned}
$$

This equation implies

$$
\overline{\boldsymbol{C}}_{n+1}^{\mathrm{alg}}=v_{\mathrm{I}}\left(\overline{\boldsymbol{C}}_{\mathrm{I}}^{\mathrm{S}}+\frac{\partial \overline{\boldsymbol{C}}_{\mathrm{I}}^{\mathrm{S}}}{\partial \bar{\varepsilon}_{\mathrm{I}}}: \Delta \varepsilon_{\mathrm{I}}^{\mathrm{r}}\right): \frac{\partial \varepsilon_{\mathrm{I}}}{\partial \bar{\varepsilon}}+v_{0}\left(\overline{\boldsymbol{C}}_{0}^{\mathrm{S}}+\frac{\partial \overline{\boldsymbol{C}}_{0}^{\mathrm{S}}}{\partial \varepsilon_{0}}: \Delta \varepsilon_{0}^{\mathrm{r}}\right): \frac{\partial \varepsilon_{0}}{\partial \bar{\varepsilon}},
$$

with $\frac{\partial \varepsilon_{\mathrm{I}}}{\partial \overline{\boldsymbol{\varepsilon}}}$ and $\frac{\partial \boldsymbol{\varepsilon}_{0}}{\partial \overline{\boldsymbol{\varepsilon}}}$ reported in Appendix C.

- An unloading step is thus applied here to fit the incremental-secant process, and the obtained results will be kept as internal variables at time interval $t_{n+1}$. The system of Eqs. (37-39), expressed at time $t_{n+1}$ instead of time $t_{n}$, has thus to be solved. The needed residual variables from unloading are the residual strains in the composite material $\bar{\varepsilon}_{n+1}^{\text {res }}$, in the inclusions phase $\varepsilon_{\mathrm{I}}^{\text {res }}{ }_{n+1}$ and in the matrix phase $\varepsilon_{0}^{\text {res }}{ }_{n+1}$, as well as the residual stresses in the fibers phase $\boldsymbol{\sigma}_{\mathrm{I}}^{\text {res }}{ }_{n+1}$ and in the matrix phase $\boldsymbol{\sigma}_{0}^{\text {res }}{ }_{n+1}$, respectively.

\footnotetext{
${ }^{4}$ In this paper we will use the term "consistent" operator for the derivative of the stress state with respect to the deformation increment $\overline{\boldsymbol{C}}^{\text {alg }}{ }_{n+1}=\frac{\partial \overline{\boldsymbol{\sigma}}_{n+1}}{\partial \Delta \boldsymbol{\varepsilon}_{\mathrm{I}_{n+1}}}$.
} 
1. The residual strain $\bar{\varepsilon}_{n+1}^{\text {res }}$ (the strain at $\overline{\boldsymbol{\sigma}}_{n+1}^{\text {res }}=0$ ) of the composite material is calculated from an unloading step, which is assumed to be a purely elastic process. The unloading operator of the composite is

$$
\begin{aligned}
\overline{\boldsymbol{C}}_{n+1}^{\mathrm{el}} & =\left[v_{\mathrm{I}} \boldsymbol{C}_{\mathrm{I} n+1}^{\mathrm{el}}: \boldsymbol{B}^{\epsilon}+v_{0} \boldsymbol{C}_{0 n+1}^{\mathrm{el}}\right]:\left[v_{\mathrm{I}} \boldsymbol{B}^{\epsilon}+v_{0} \boldsymbol{I}\right]^{-1} \\
\text { with } \quad \boldsymbol{B}^{\epsilon} & =\left\{\boldsymbol{I}+\boldsymbol{S}:\left[\left(\boldsymbol{C}_{0 n+1}^{\mathrm{el}}\right)^{-1}: \boldsymbol{C}_{\mathrm{I} n+1}^{\mathrm{el}}-\boldsymbol{I}\right]\right\}^{-1}
\end{aligned}
$$

Note that $\boldsymbol{C}_{0 n+1}^{\mathrm{el}}$ is used in the Eshelby tensor of the elastic unloading step. The residual strain of the composite can be calculated by

$$
\begin{aligned}
\overline{\boldsymbol{\varepsilon}}_{n+1}^{\mathrm{res}} & =\overline{\boldsymbol{\varepsilon}}_{n+1}-\Delta \overline{\boldsymbol{\varepsilon}}_{n+1}^{\text {unload }} \\
& =\overline{\boldsymbol{\varepsilon}}_{n+1}-\left(\overline{\boldsymbol{C}}_{n+1}^{\mathrm{el}}\right)^{-1}: \overline{\boldsymbol{\sigma}}_{n+1} .
\end{aligned}
$$

2. The residual strains in the fibers and in the matrix phases are computed following the M-T method, which gives:

$$
\begin{aligned}
\varepsilon_{\mathrm{I}{ }_{n+1}}^{\text {res }} & =\boldsymbol{\varepsilon}_{\mathrm{I} n+1}-\Delta \boldsymbol{\varepsilon}_{\mathrm{I}}^{\text {unload }}{ }_{n+1} \\
& =\boldsymbol{\varepsilon}_{\mathrm{I} n+1}-\boldsymbol{B}^{\epsilon}:\left[v_{\mathrm{I}} \boldsymbol{B}^{\epsilon}+v_{0} \boldsymbol{I}\right]^{-1}: \Delta \overline{\boldsymbol{\varepsilon}}_{n+1}^{\text {unload }}, \\
\varepsilon_{0}^{\mathrm{res}}{ }_{n+1} & =\varepsilon_{0 n+1}-\Delta \varepsilon_{0}^{\text {unload }}{ }_{n+1} \\
& =\boldsymbol{\varepsilon}_{0 n+1}-\left[v_{\mathrm{I}} \boldsymbol{B}^{\epsilon}+v_{0} \boldsymbol{I}\right]^{-1}: \Delta \overline{\boldsymbol{\varepsilon}}_{n+1}^{\text {unload }} .
\end{aligned}
$$

3. The residual stresses in the fibers and matrix phases can be obtained, respectively, from

$$
\begin{aligned}
& \sigma_{\mathrm{I} n+1}^{\text {res }}=\sigma_{\mathrm{I} n+1}-C_{\mathrm{I} n+1}^{\mathrm{el}}: \Delta \varepsilon_{\mathrm{I}}^{\text {unload }}{ }_{n+1}, \\
& \sigma_{0 n+1}^{\text {res }}=\sigma_{0 n+1}-C_{0 n+1}^{\text {el }}: \Delta \varepsilon_{0}^{\text {unload }}{ }_{n+1} .
\end{aligned}
$$

From this detailed incremental-secant MFH process, it is clear that the method can be implemented at the material law level of a FE code. Moreover this homogenized material law calls successively the constitutive models of both the inclusions and the matrix phases. The constitutive laws of these two materials are the usual elasto-plastic models at two exceptions: the modification of the return mapping direction and the computation, as an output of the material model, of the secant tensor $C^{\mathrm{S}}$. For an elastic phase, no modification is required. The incremental-secant $\mathrm{MFH}$ is thus simple to implement and is computationally efficient as the phases constitutive material models remain classical and independent from each-other. Note that considering a second-moment method involves deeper modifications of the material models of the different composite material phases. 


\section{Comparison with direct finite element simulations, fast-Fourier transforms methods or experimental results}

In this section, the accuracy and the reliability of the proposed incrementalsecant method are verified through the comparison of the effective response of several two-phase elasto-plastic composites. Reference results are provided either by direct FE simulations on representative cells of the micro-structure, or by experimental data found in the literature. All the MFH results presented have converged with the size of the strain increments.

First we study the difference of results obtained by considering the residualincremental-secant method or the zero-incremental-secant method for the elasto-plastic matrix phase of composites reinforced by elastic inclusions. Note that for elastic phases the incremental-secant operator is the elastic operator, as the elastic phase has no need for a virtual elastic property to be defined. It will be shown that considering the zero-incremental method in the elasto-plastic phase improves the results accuracy. Then the method is shown to predict results as accurate as other MFH methods, including the incremental-tangent method and the variational method, on a wide variety of composite materials made of an elasto-plastic matrix reinforced by elastic inclusions. Finally the effect of the incremental-secant operator choice for composites made of two elasto-plastic phases is studied.

\subsection{Predictions based on the two different definitions of the incremental- secant moduli $-\boldsymbol{C}^{S r}$ and $\boldsymbol{C}^{S O}$ - for composite materials made of an elasto- plastic matrix reinforced by elastic inclusions}

In section 3.1, we proposed two different definitions of the incrementalsecant moduli: $\boldsymbol{C}^{\mathrm{Sr}}$ and $\boldsymbol{C}^{\mathrm{S} 0}$ that can be used to define the LCC. The effects of the different definitions on the predictions are studied herein through a few numerical examples, which are carried out on some composites with elastic inclusions.

In this case, since the inclusions are made of elastic materials, this phase has no need for a virtual elastic property to be defined. We will thus study the effect of the operator choice for the matrix phase on several examples to confirm that the zero-incremental-secant operator $C_{0}^{\mathrm{S} 0}$ should be used for the latter.

Metal Matrix Composites (MMCs) with elastic inclusions and elastic-perfectlyplastic matrix. An elastic-perfectly-plastic behavior for the matrix phase is 


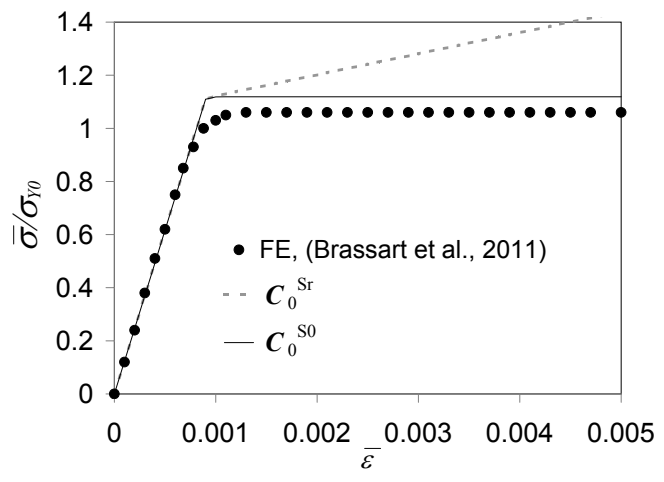

Figure 4: Results for composites with elastic inclusions and elastic-perfectly-plastic matrix.

considered $\left(R_{0}(p)=0\right)$ while the inclusions remain elastic. The other material properties are

- Inclusions: $E_{\mathrm{I}}=400 \mathrm{GPa}, \nu_{\mathrm{I}}=0.2$;

- Matrix: $E_{0}=75 \mathrm{GPa}, \nu_{0}=0.3, \sigma_{Y 0}=75 \mathrm{MPa}$.

Inclusions are spherical with a volume fraction of $v_{\mathrm{I}}=15 \%$. The predictions with the two incremental-secant moduli for the matrix phase, together with FE reference results provided by Brassart et al. (2011), are presented in Fig. 4, which shows that the zero-incremental-secant method should be used for the matrix to agree with the FE results.

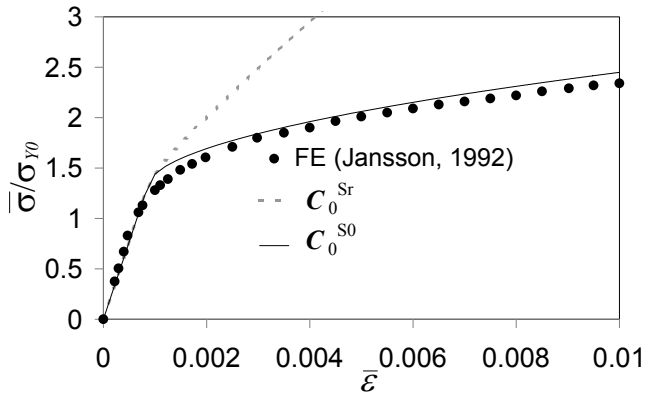

(a) Transverse loading

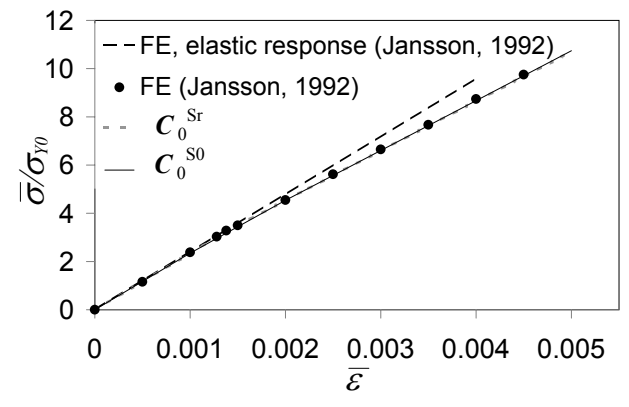

(b) Longitudinal loading

Figure 5: Comparisons of the secant-incremental methods for Metal Matrix Composites with elastic fibers embedded in an elasto-plastic matrix. 
MMCs with elastic inclusions and elasto-plastic matrix. The example consists of an aluminum alloy matrix reinforced with continuous stiff alumina fibers, whose material properties are (Doghri and Friebel, 2005)

- Inclusions: $E_{\mathrm{I}}=344.5 \mathrm{GPa}, \nu_{\mathrm{I}}=0.26$;

- Matrix: $E_{0}=68.9 \mathrm{GPa}, \nu_{0}=0.32, \sigma_{Y 0}=94 \mathrm{MPa}, k_{0}=578.25 \mathrm{MPa}$ and $m_{0}=0.529$.

The matrix material follows the hardening law

$$
R(p)=k p^{m},
$$

where $k$ and $m$ are the hardening parameters. The volume fraction of the continuous fibers is $v_{\mathrm{I}}=55 \%$. The $\mathrm{FE}$ predictions were obtained by Jansson (1992) on a unit cell assuming a periodic microstructure with a hexagonal arrangement. The MFH results for the two incremental-secant moduli for the matrix phase are presented in Fig. 5(a) for a transerve loading, and in Fig. 5(b) for a longitudinal loading.

For the transverse loading, see Fig. 5(a), stiffer results are obtained when using $\boldsymbol{C}_{0}^{\mathrm{Sr}}$, while good predictions are obtained when using $\boldsymbol{C}_{0}^{\mathrm{S} 0}$. Good predictions are observed with both operators for the longitudinal loading, see Fig. 5(b).

The reason why using the zero-incremental-secant formulation for the elasto-plastic phase improves the results accuracy is the following. As only the matrix phase exhibits a plastic flow, upon the virtual unloading the residual stress in the matrix is negative (assuming the composite material was under tension and vice versa), while the residual stress in the inclusions phase is positive. Using the zero-incremental-secant operator, meaning neglecting the residual stress, in the matrix induces thus more plasticity as the predictor is higher, which counterbalances the over-stiff results predicted by a firstmoment method as the one proposed herein. We think that considering a second-moment approach would solve this issue.

We will show in the next section that for problems with elastic inclusions, using the zero-incremental-secant approach $\boldsymbol{C}_{0}^{\mathrm{S0}}$ for the elasto-plastic matrix phase leads to a good accuracy for different materials, inclusion geometries and loading path. 
4.2. Predictions for elastic inclusions and with the zero-incremental-secant operator $C_{0}^{S O}$ for the elasto-plastic matrix

In this section, the considered composites consist in an elasto-plastic matrix reinforced by ellipsoidal or continuous UD inclusions with linear elastic behaviors. The inclusions remain elastic and the matrix phase obeys to the zero-incremental-secant formulation. The predictions of the proposed model are compared to references provided either by direct FE simulations on representative cells of the micro-structure or by experimental data gathered from literature.

\subsubsection{Responses for short and UD fibers reinforced matrix}

The method is here applied to composites with short and UD fibers. In particular, the response in the phases is compared to reference results.

GFRP: Short glass fibers reinforced polyamide. In this case, the polyamide matrix follows an exponential-linear hardening law:

$$
R(p)=k_{1} p+k_{2}\left(1-e^{-m p}\right)
$$

and the material properties are

- Inclusions: $E_{\mathrm{I}}=72 \mathrm{GPa}, \nu_{\mathrm{I}}=0.22$;

- Matrix: $E_{0}=2.1 \mathrm{GPa}, \nu_{0}=0.3, \sigma_{Y 0}=29 \mathrm{MPa}, k_{10}=139.0 \mathrm{MPa}$, $k_{20}=32.7 \mathrm{MPa}$ and $m_{0}=319.4$.

The glass fibers volume fraction is $v_{\mathrm{I}}=15.7 \%$ and their aspect ratio is $\alpha=15$. Aligned fibers are considered and the FE simulations were performed by Doghri et al. (2011). These FE results are used as reference. Fig. 6(a) shows the macroscopic predictions of the two MFH formulations under longitudinal (along the fibers direction) uni-axial tension, and Fig. 6(b) shows the ones under transverse uni-axial tension. Results reported by Doghri et al. (2011) using first-moment and second-moment incrementaltangent methods are also reported for comparison purposes. On the one hand, for a transverse loading all the MFH methods predict accurate results, see Fig. 6(b). On the other hand, for a longitudinal loading, although the second-moment incremental-tangent method predicts a response less stiff than the first-moment schemes, the new scheme exhibits a better accuracy when compared to the first-moment incremental-tangent method, see Fig. 
6(a). When analyzing the average von Mises stress predicted in the matrix phase $^{5}$, the predictions are really accurate for a transverse loading, see Fig. 6(d). For a loading aligned in the fibers direction, see Fig. 6(c), the newly developed method is as accurate as the results obtained with the secondmoment incremental-tangent method. This behavior is confirmed by the evolution of the average accumulated plastic strain in the matrix phase as shown by Figs. 6(e) and 6(f).

Continuous UD-fibers reinforced elasto-plastic matrix. The accuracy of the proposed incremental-secant method is assessed through the comparison with direct FE simulations on periodical cells of a continuous UD fibers reinforced elasto-plastic matrix. This consists of continuous elastic isotropic fibers embedded in a matrix material, which follows a classical J2-elastoplastic behavior model. The material parameters, taken from (Wu et al., 2012), are

- Inclusions: $E_{\mathrm{I}}=238 \mathrm{GPa}, \nu_{\mathrm{I}}=0.26$;

- Matrix: $E_{0}=2.89 \mathrm{GPa}, \nu_{0}=0.3, \sigma_{Y 0}=35 \mathrm{MPa}, k_{0}=73 \mathrm{MPa}, m_{0}=60$.

The matrix material behavior follows the hardening law

$$
R(p)=k\left(1-e^{-m p}\right)
$$

The volume fraction of the continuous fibers is $v_{\mathrm{I}}=50 \%$. The test consists of a transverse loading of the composite, followed by a complete unloading until reaching a zero-strain state. In the direction of the fibers, plane-strain conditions are assumed, while plane-stress conditions are applied in the other transverse direction.

Figure 7(a) compares the macro-stress evolution obtained with the new incremental-secant $\mathrm{MFH}$ and the incremental-tangent $\mathrm{MFH}$ to the FE results. During the loading part, the incremental-secant method avoids the over-stiff prediction exhibited by the incremental-tangent method. This is confirmed when analyzing the evolution of the average stress in the inclusions phase, see Fig. 7(b), and in the matrix phase, see Fig. 7(c). However, during

\footnotetext{
${ }^{5}$ For the second-moment method, both the average of the equivalent von Mises stress, and the von Mises stress computed from the average stress tensor in the matrix are reported.
} 


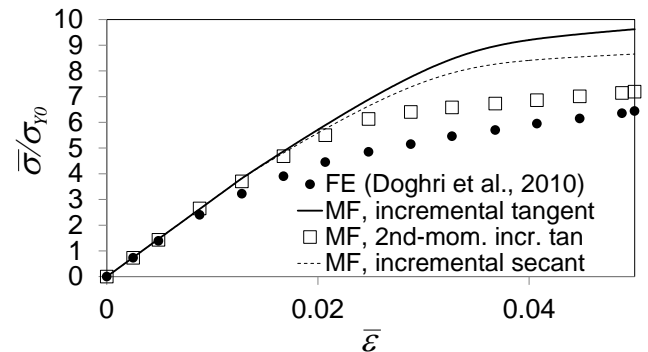

(a) $\bar{\sigma}$, Longitudinal loading

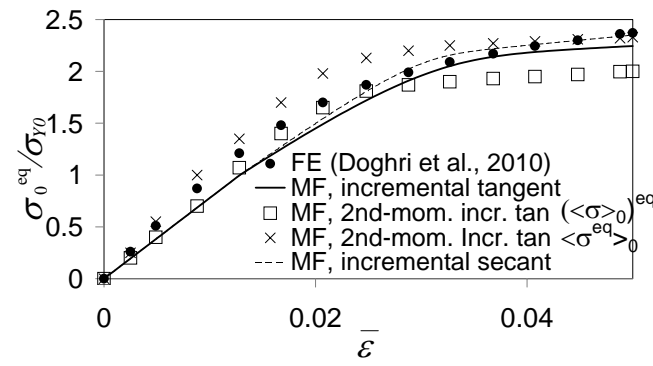

(c) $\sigma_{0}^{\text {eq }}$, Longitudinal loading

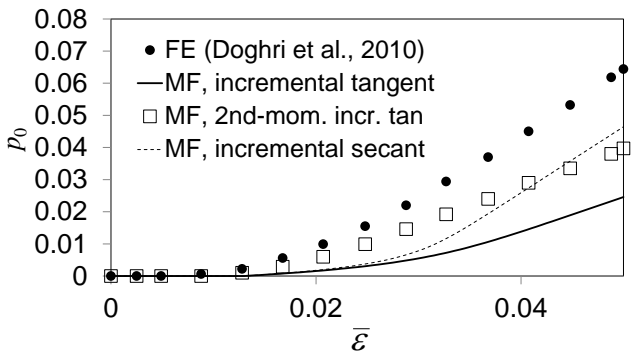

(e) $p_{0}$, Longitudinal loading

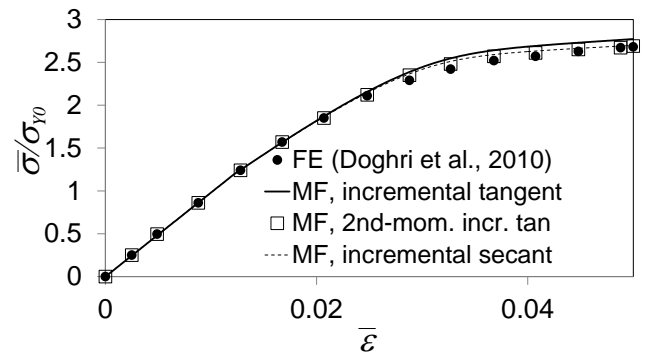

(b) $\bar{\sigma}$, Transverse loading

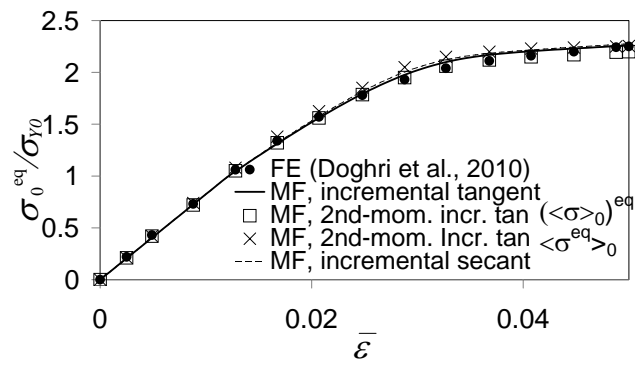

(d) $\sigma_{0}^{\text {eq }}$, Transverse loading

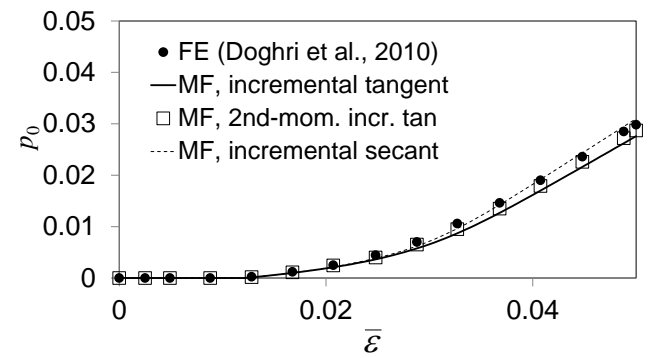

(f) $p_{0}$, Transverse loading

Figure 6: Results for the short glass fibers reinforced polyamide test for (a, c, e) a longitudinal traction and for (b, d, f) a transverse traction. (a-b) Composite response, (c-d) evolution of the average of the von Mises stress in the matrix phase, (e-f) evolution of the accumulated plastic strain in the matrix phase.

the unloading part, the stress is slightly lower than the one predicted by the FE simulation and by the incremental-tangent method. This last method exhibits less error as it starts from a higher stress at the unloading point, so this compensates for the error due to an over-stiff response. Figure 7(d) compares the average value of the effective equivalent von Mises stress in the matrix and Fig. 7(e) illustrates the accumulated plastic strain evolution in 


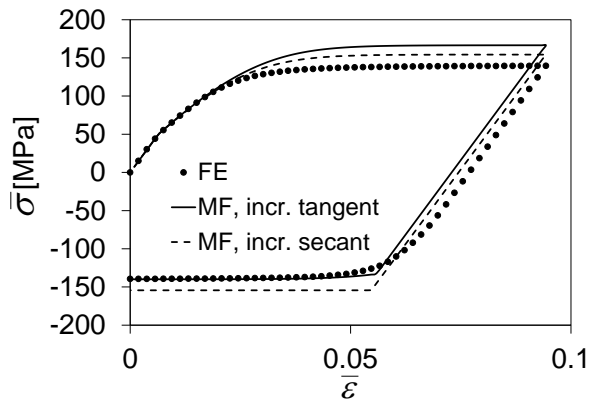

(a) $\bar{\sigma}$ vs. $\bar{\varepsilon}$

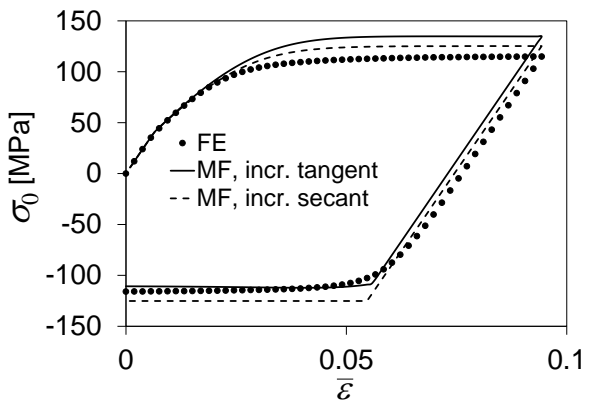

(c) $\sigma_{0}$ vs. $\bar{\varepsilon}$

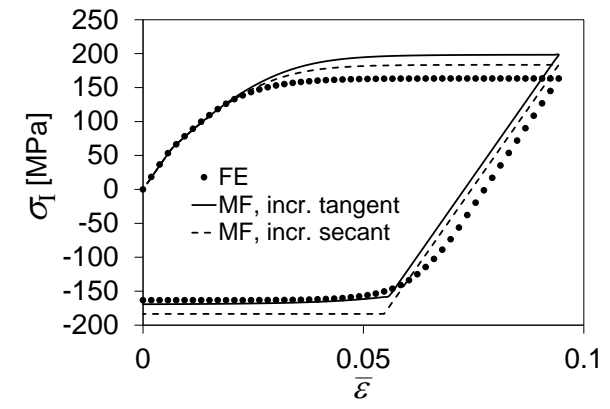

(b) $\sigma_{\text {I }} v s \cdot \bar{\varepsilon}$

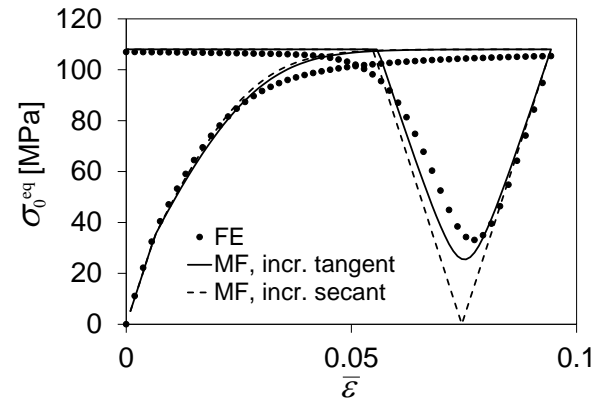

(d) $\sigma_{0}^{\text {eq }}$ vs. $\bar{\varepsilon}$

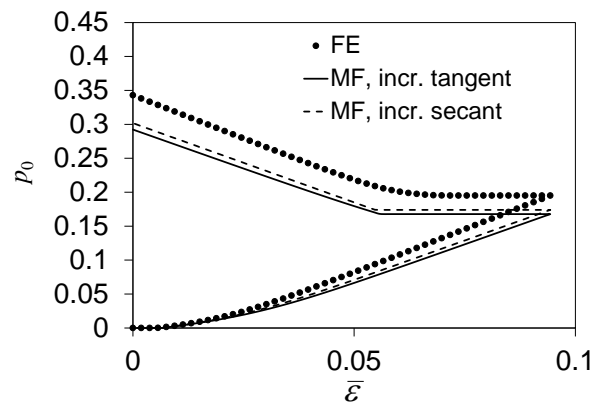

(e) $p_{0}$ vs. $\bar{\varepsilon}$

Figure 7: Results for continuous-elastic fibers embedded in an elasto-plastic matrix under transverse tension-compression.

the matrix. Both incremental methods are found to give similar results.

\subsubsection{Metal Matrix Composites (MMCs)}

Now, the proposed incremental-secant procedure is applied on various Metal Matrix Composites (MMCs), which are made of an elasto-plastic matrix reinforced by elastic inclusions. The matrix behavior follows the power- 
law hardening (53).

$M M C \# 1$ : an aluminum alloy reinforced with stiff ceramic particles I. The properties of the considered material are

- Inclusions: $E_{\mathrm{I}}=400 \mathrm{GPa}, \nu_{\mathrm{I}}=0.2$;

- Matrix: $E_{0}=75 \mathrm{GPa}, \nu_{0}=0.3, \sigma_{Y 0}=75 \mathrm{MPa}, k_{0}=416 \mathrm{MPa}$ and $m_{0}=0.3895$.

Inclusions are spherical with a volume fraction of $v_{\mathrm{I}}=30 \%$. The macroscopic prediction obtained with the incremental-secant and the first order incremental-tangent formulations are presented in Fig. 8(a) together with FE predictions obtained on a random arrangement of 30 inclusions reported by Segurado et al. (2002).

MMC \#2: an aluminum alloy reinforced with stiff ceramic particles II. The same material system as in case $\mathrm{MMC \# 1}$ is considered here again, except for the hardening law. The initial yield stress $\sigma_{Y 0}$ is set equal to zero for the computation and the hardening exponents $m_{0}=0.05$ and $m_{0}=0.4$ of the matrix material are considered successively. The inclusions are aligned ellipsoids with an aspect ratio $\alpha=3$ and the volume fraction of the inclusions is $v_{\mathrm{I}}=25 \%$. These material properties correspond to the composites studied by Pierard et al. (2007a), who provided the FE results on unit cells containing 30 inclusions. A uni-axial tension is carried out in the longitudinal direction of the ellipsoidal inclusions. The results of the two MFH formulations together with the FE results provided by Pierard et al. (2007a) are presented in Fig. 8(b) for $m_{0}=0.05$, and in Fig. 8(c) for $m_{0}=0.4$, respectively.

$M M C$ \#3: an aluminum alloy reinforced with SiC whiskers. The properties of the considered material are

- Inclusions: $E_{\mathrm{I}}=450 \mathrm{GPa}, \nu_{\mathrm{I}}=0.17$;

- Matrix: $E_{0}=68.89 \mathrm{GPa}, \nu_{0}=0.33, \sigma_{Y 0}=277.3 \mathrm{MPa}, k_{0}=592.2 \mathrm{MPa}$ and $m_{0}=0.52$.

The elastic properties of the whiskers were reported by Christman et al. (1989a) and the properties of the matrix were fitted by Doghri and Friebel (2005) on the experimental data reported by Christman et al. (1989b). The volume fraction of the inclusions is $v_{\mathrm{I}}=13.2 \%$. The whiskers are assumed 
to be cylindrical with the length-diameter ratio $l / d$ of 5 . For the $\mathrm{MFH}$ computations, the aspect ratio $\alpha$ of the equivalent ellipsoid is obtained by multiplying $l / d$ with a factor of 1.25 , as proposed by Li and Ponte Castañeda (1994) and Doghri and Friebel (2005).

The experimental data for a uni-axial tension applied along the longitudinal direction of the whiskers are reported by Christman et al. (1989a,b), and the stress-strain curves are presented in Fig. $8(\mathrm{~d})$ together with the MFH results. This figure also represents the curve-fitting of the matrix material considered.

$M M C \#$ 4: a two-phase steel made of ellipsoidal martensitic inclusions embedded in a ferritic matrix. The properties of the two phases are

- Inclusions: $E_{\mathrm{I}}=200 \mathrm{GPa}, \nu_{\mathrm{I}}=0.3$;

- Matrix: $E_{0}=220 \mathrm{GPa}, \nu_{0}=0.3, \sigma_{Y 0}=300 \mathrm{MPa}, k_{0}=1130 \mathrm{MPa}$ and $m_{0}=0.31$.

The volume fraction of the inclusions is $v_{\mathrm{I}}=25 \%$. The inclusions are successively considered as spherical, $\alpha=1$, and as ellipsoidal with $\alpha=3$ and the results are reported in Fig.8(e) and in Fig.8(f) respectively. These figures show the comparison of the two MFH predictions with the FE results, which reference results were obtained by Brassart et al. (2010) on multiparticle cells.

Fig. 8 shows that the proposed MFH method predicts responses slightly more compliant than with the incremental-tangent method. However the accuracy can be said to be of the same order.

\subsubsection{Effect of the triaxiality}

In this section, more general loading conditions are applied to test the reliability of the proposed method. We applied a pure shear loading, a biaxial loading and a plane strain tension/compression successively on a MMC to investigate the effect of triaxiality on the accuracy of the predictions. The considered composite is a $\mathrm{SiC}$-particles reinforced aluminum matrix (MMC \#5). The elasto-plastic metal matrix follows the power-law hardening (53) and the material properties are

- Inclusions: $E_{\mathrm{I}}=400 \mathrm{GPa}, \nu_{\mathrm{I}}=0.2$;

- Matrix: $E_{0}=75 \mathrm{GPa}, \nu_{0}=0.3, \sigma_{Y 0}=75 \mathrm{MPa}, k_{0}=400 \mathrm{MPa}$ and $m_{0}=0.4$ or $m_{0}=0.05$. 


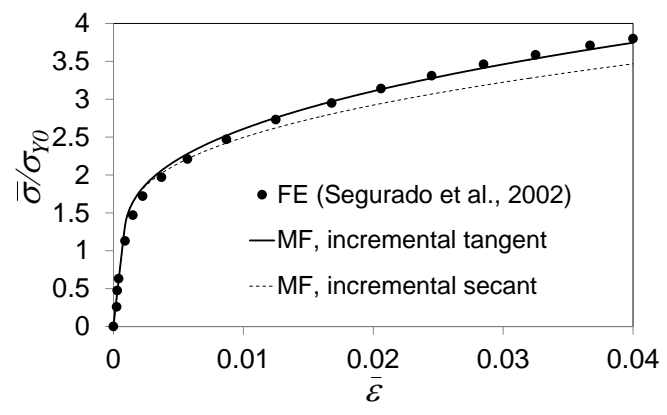

(a) MMC \#1

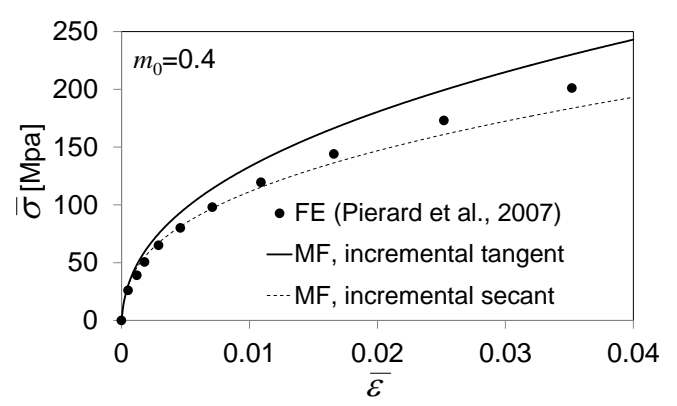

(c) $\mathrm{MMC} \# 2, m_{0}=0.4$

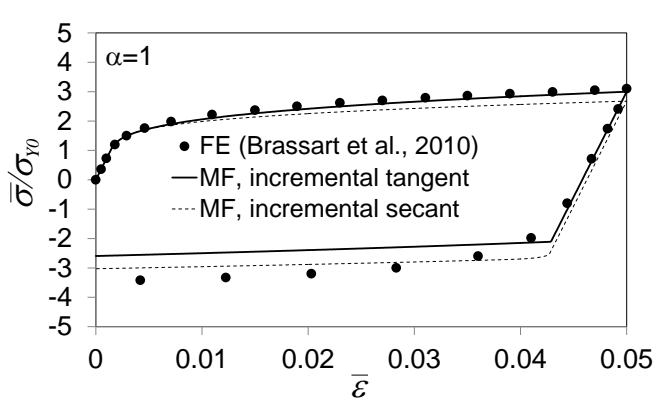

(e) $\mathrm{MMC} \# 4, \alpha=1$

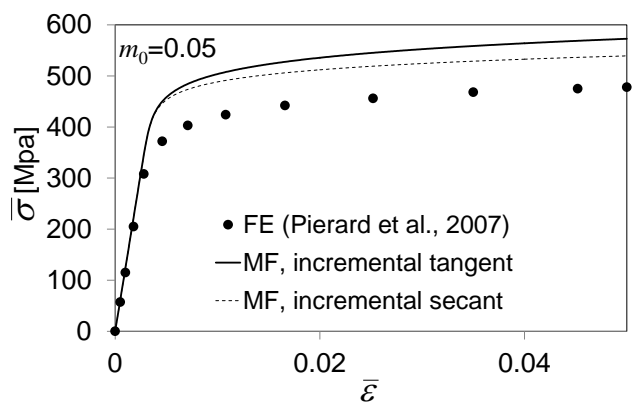

(b) $\mathrm{MMC} \# 2, m_{0}=0.05$

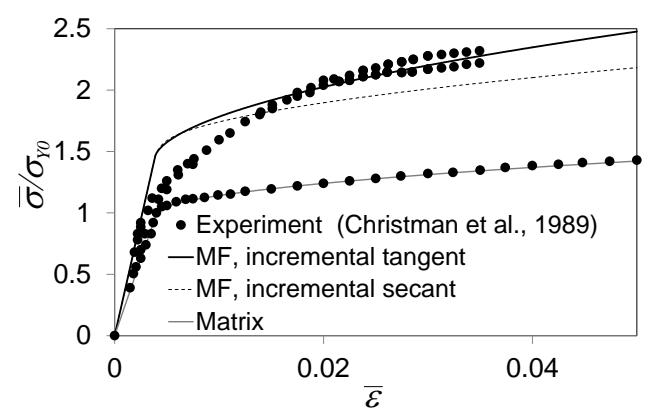

(d) MMC \#3

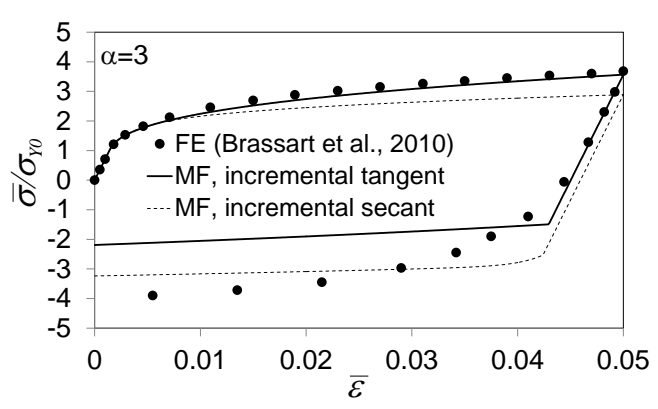

(f) $\mathrm{MMC} \# 4, \alpha=3$

Figure 8: Results for the various metal matrix composites tests.

The volume fraction of the spherical inclusions is $v_{\mathrm{I}}=15 \%$. The predictions of the incremental secant formulation are presented in Fig. 9. The reference macro-responses are direct finite element results presented by Brassart et al. (2012), which are obtained on periodic cells containing 35 randomly dispersed inclusions. The mean field homogenization predictions obtained by the firstmoment incremental-tangent method and by the variational method are also 


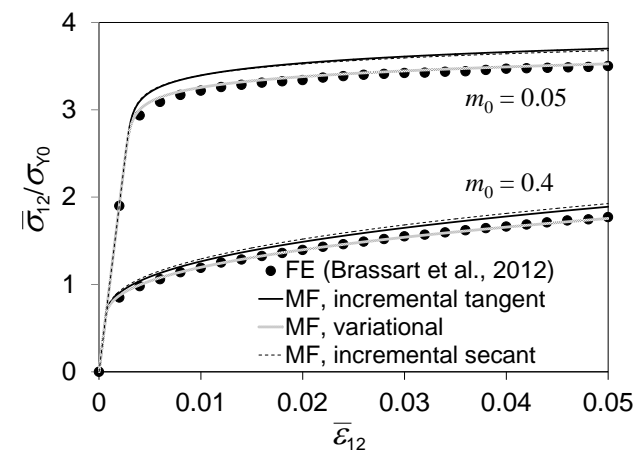

(a) Shearing

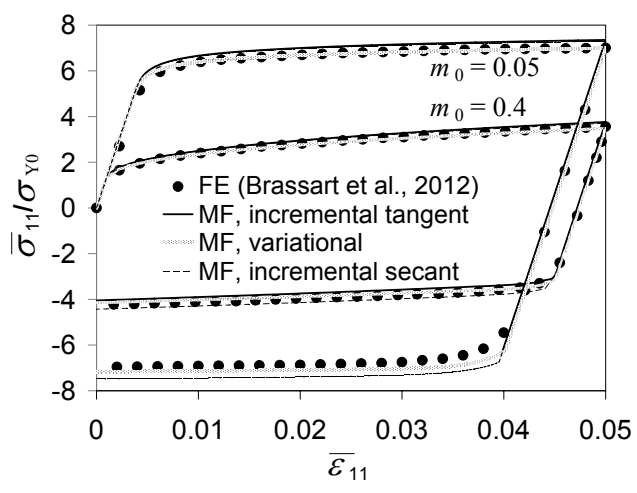

(c) Tension-compression,

11-Loading stress, methods comparison

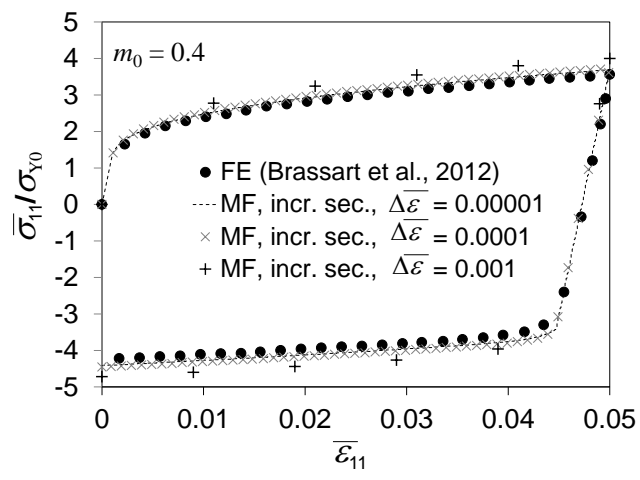

(e) Tension-compression, 11-Loading stress, strain increment size effect

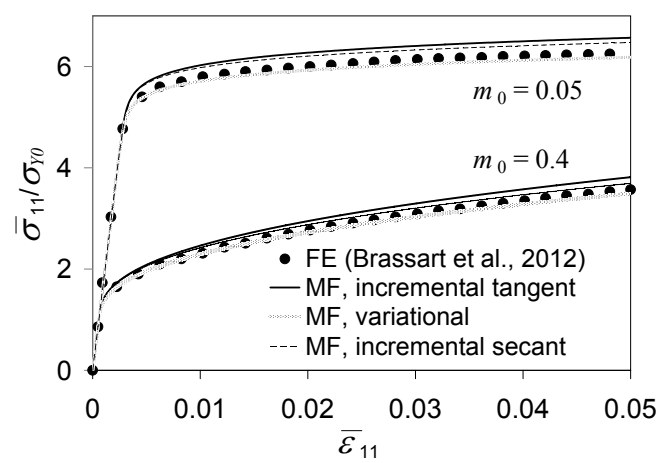

(b) Biaxial tension

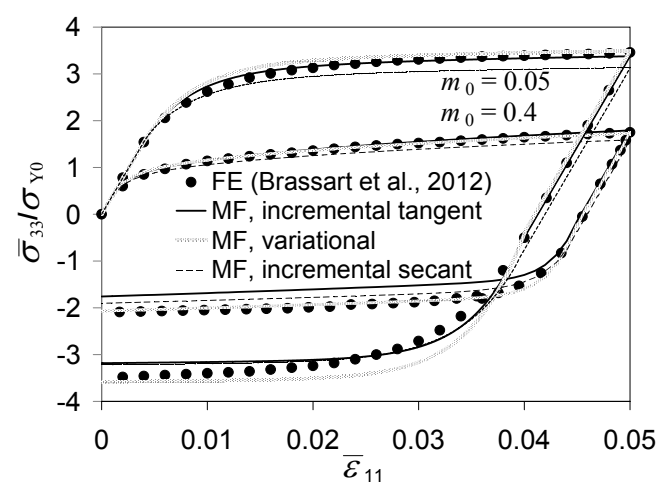

(d) Tension-compression, 33-Reaction stress, methods comparison

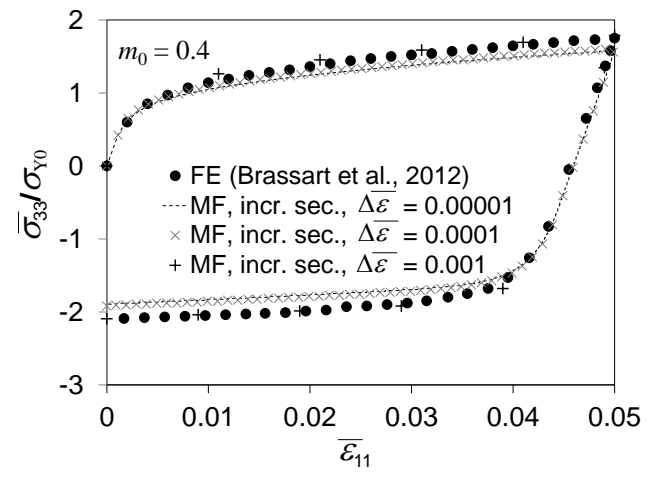

(f) Tension-compression, 33-Reaction stress, strain increment size effect

Figure 9: Results for MMC \#5 under different loading conditions. 
presented in Fig. 9 for comparison.

For this problem, three different loading conditions are successively applied. First, shear loading, with $\bar{\sigma}_{12}=\bar{\sigma}_{21}=\sigma$ and the other components of the stress tensor $\overline{\boldsymbol{\sigma}}$ being zero, is considered. Then, a biaxial loading is obtained by setting $\bar{\sigma}_{11}=\bar{\sigma}_{22}=\sigma$ and $\bar{\sigma}_{33}=0$. Finally, in the case of plane strain tension/compression, the only non-zero components of $\bar{\varepsilon}$ are $\bar{\varepsilon}_{11}$ and $\bar{\varepsilon}_{22}$, with $\bar{\varepsilon}_{22}$ computed to satisfy $\bar{\sigma}_{22}=0$. Moreover $\bar{\varepsilon}_{33}$ is set to zero, and $\bar{\sigma}_{33}$ can be measured.

These different loading conditions correspond to different triaxiality states. Indeed, as the macroscopic triaxiality ratio is defined by $T=\operatorname{tr}(\overline{\boldsymbol{\sigma}}) / 3 \bar{\sigma}^{e q}$, one can directly find that shear and biaxial loading conditions correspond to triaxiality ratios of 0 and $2 / 3$ respectively. The uniaxial tension, which has been applied a lot in the previous examples, has a triaxiality ratio of $1 / 3$. For plane strain tension/compression, the triaxiality ratio is approximately 1 in the plastic regime.

In Fig, 9, the predictions of the proposed method shows an accuracy comparable to the other MFH approaches, for the different triaxiality states. Moreover, the effect of the strain increment size on the results predicted by the new incremental-secant method has been studied in Figs. 9(e) and 9 (f) for the last test with the hardening exponent $m_{0}=0.4$. It can be seen that the results have already converged for a macro-strain increment of $\Delta \bar{\varepsilon}=0.0001$.

\subsubsection{Non-monotonic, non-proportional loading path}

Eventually the behavior of the incremental-secant MFH is tested with a non-monotonic, non-proportional loading path. This example was proposed by Lahellec and Suquet (2013) and consists of elastic spherical inclusions embedded in an elastic-perfectly-plastic matrix, with the following material properties

- Inclusions: $\kappa_{\mathrm{I}}^{\mathrm{el}}=20 \mathrm{GPa}, \mu_{\mathrm{I}}^{\mathrm{el}}=6 \mathrm{GPa}$;

- Matrix: $\kappa_{0}^{\mathrm{el}}=10 \mathrm{GPa}, \mu_{0}^{\mathrm{el}}=3 \mathrm{GPa}, \sigma_{Y 0}=100 \mathrm{MPa}$.

The volume fraction of the spherical inclusions is $v_{\mathrm{I}}=17 \%$. The external boundary conditions correspond to constraining simultaneously all the strain 


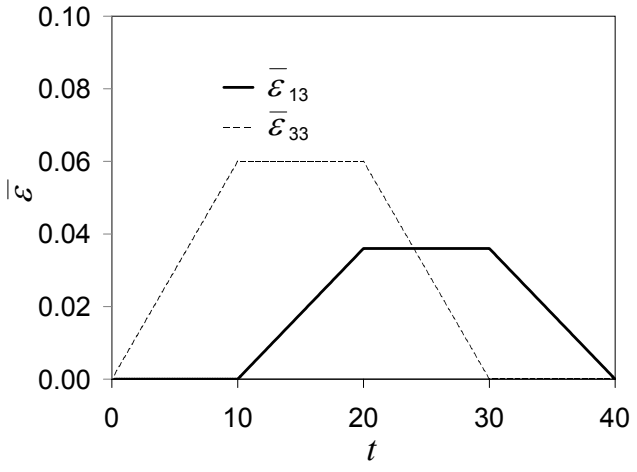

(a) Loading path

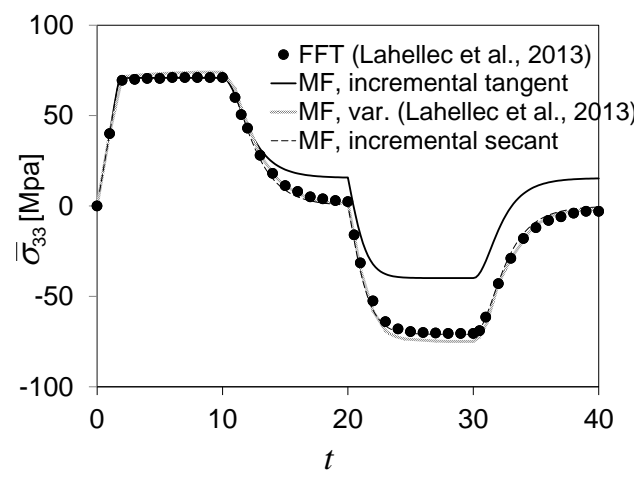

(c) Tensile response

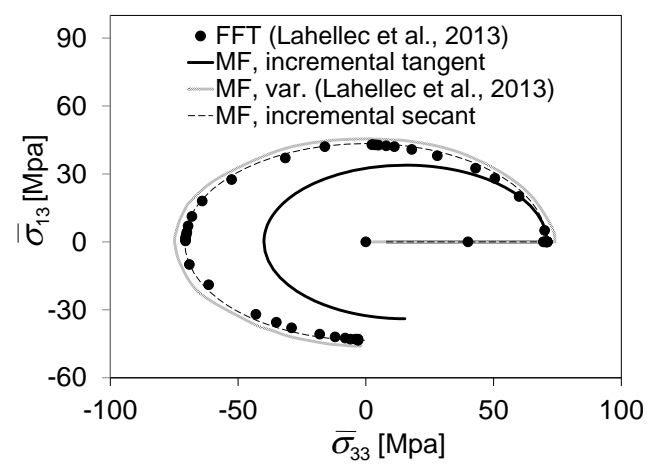

(b) Stress components

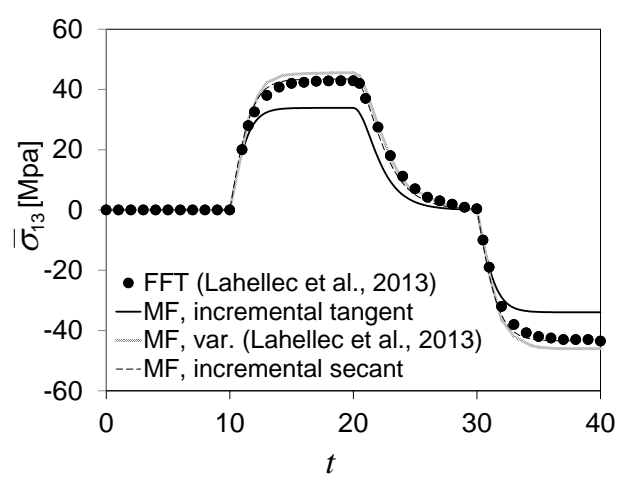

(d) Shear response

Figure 10: Results for a non-monotonic, non-proportional loading path. (a) Applied strain components history (b) Comparisons of the predicted stress components (c) Comparison of the predicted stress components history.

components following

$$
\begin{aligned}
\overline{\boldsymbol{\varepsilon}}(t)= & \bar{\varepsilon}_{33}(t)\left[\boldsymbol{e}_{3} \otimes \boldsymbol{e}_{3}-\frac{1}{2}\left(\boldsymbol{e}_{1} \otimes \boldsymbol{e}_{1}+\boldsymbol{e}_{2} \otimes \boldsymbol{e}_{2}\right)\right]+ \\
& \bar{\varepsilon}_{13}(t)\left(\boldsymbol{e}_{1} \otimes \boldsymbol{e}_{3}+\boldsymbol{e}_{3} \otimes \boldsymbol{e}_{1}+\boldsymbol{e}_{2} \otimes \boldsymbol{e}_{3}+\boldsymbol{e}_{3} \otimes \boldsymbol{e}_{2}\right),
\end{aligned}
$$

where the non-monotonic, non-proportional evolution of the two constraints with respect to a fictitious time is illustrated in Fig. 10(a).

This problem was solved using a Fast Fourier Transforms (FFT)-based numerical method and the variational MFH by Lahellec and Suquet (2013), and the resulting tensile and shear response components are reported in Fig. 10. The new incremental-secant and the incremental-tangent MFH schemes are then applied. The results are illustrated in Figs. 10(b), 10(c) and 
10(d). It can be seen that in this test the incremental-secant formulation has an accuracy comparable to the variational method, while the incrementaltangent method is unable to capture the change of loading direction.

\subsection{Elasto-plastic inclusions reinforced elasto-plastic matrix}

We have shown that, for composites made of elasto-plastic matrix reinforced by elastic inclusions, considering the zero-incremental-secant approach in the matrix phase instead of the residual-incremental-secant approach improves the accuracy of the results as it counterbalances the overstiff prediction inherent to a first-moment method.

In this section the case of elasto-plastic inclusions reinforced elastoplastic matrix composites is considered. For a composite made of two elastoplastic phases, four cases according to the combinations of the different incremental-secant moduli for the inclusions and matrix phases are successively considered:

1. $C_{\mathrm{I}}^{\mathrm{Sr}}$ and $C_{0}^{\mathrm{Sr}}$,

2. $C_{\mathrm{I}}^{\mathrm{S} 0}$ and $C_{0}^{\mathrm{SO}}$,

3. $C_{\mathrm{I}}^{\mathrm{Sr}}$ and $C_{0}^{\mathrm{So}}$, and

4. $\boldsymbol{C}_{\mathrm{I}}^{\mathrm{S} 0}$ and $\boldsymbol{C}_{0}^{\mathrm{Sr}}$.

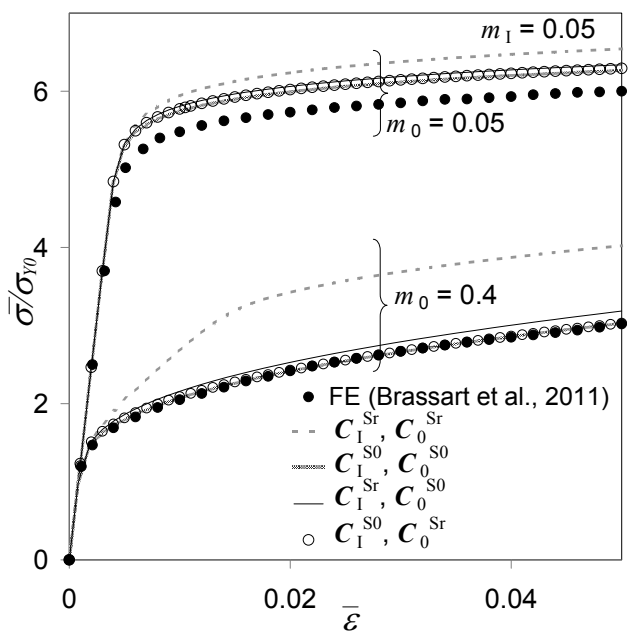

(a) $m_{\mathrm{I}}=0.05$

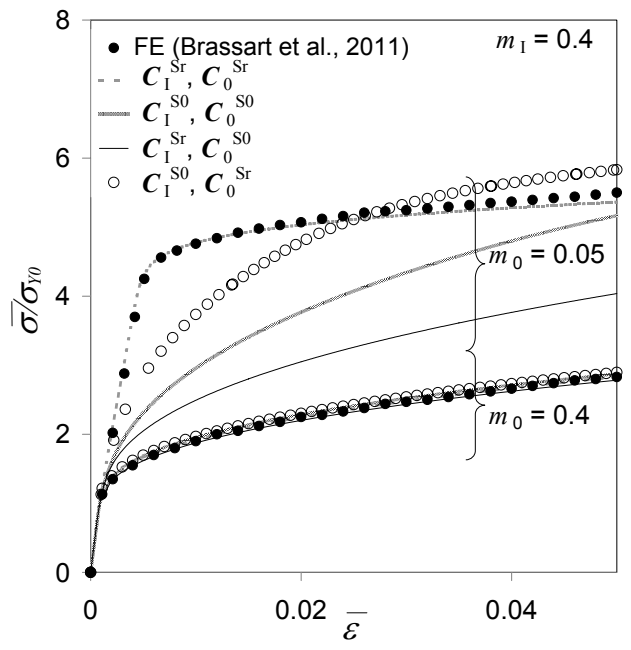

(b) $m_{\mathrm{I}}=0.4$

Figure 11: Results for the elasto-plastic inclusions - elasto-plastic matrix tests. 
Metal Matrix Composites (MMCs) with power-law hardening in both phases. In this example studied by Brassart et al. (2011), both matrix and inclusions phases obey an elasto-plastic behavior with a power-law hardening (53). The material properties for the inclusions and matrix are

- Inclusions: $E_{\mathrm{I}}=400 \mathrm{GPa}, \nu_{\mathrm{I}}=0.2, \sigma_{Y \mathrm{I}}=75 \mathrm{MPa}, k_{\mathrm{I}}=1.0 \mathrm{GPa}$, $m_{\mathrm{I}}=0.4$ or $m_{\mathrm{I}}=0.05$;

- Matrix: $E_{0}=75 \mathrm{GPa}, \nu_{0}=0.3, \sigma_{Y 0}=75 \mathrm{MPa}, k_{0}=400 \mathrm{MPa}$ and $m_{0}=0.4$ or $m_{0}=0.05$.

Inclusions are spherical with a volume fraction of $v_{\mathrm{I}}=15 \%$. Uni-axial tension tests are performed, and the macroscopic responses are predicted for the four different incremental-secant moduli cases with the newly proposed MFH formulation. The reference results are obtained from the $\mathrm{FE}$ simulations reported in section 6.2 of (Brassart et al., 2011) for periodic composites.

First, in the case of comparable hardening for the matrix and inclusions materials, $m_{0}=m_{\mathrm{I}}=0.05$ in Fig. 11(a) and $m_{0}=m_{\mathrm{I}}=0.4$ in Fig.11(b), the residual stresses remain small in both phases and satisfactory results are obtained for the four combinations although for $m_{0}=m_{\mathrm{I}}=0.05$, see Fig. 11(a), using the residual-incremental-secant operator in both matrix and inclusions phases overestimates the results slightly more than for the other combinations.

Second, in the case of a more severe hardening in the inclusions phase, $m_{0}=0.4$ and $m_{\mathrm{I}}=0.05$ in Fig. 11(a), the overestimation of the predictions when considering the residual-incremental-secant operator in both matrix and inclusions phases becomes unacceptable. The three other combinations give good predictions.

Third, in the case of a more severe hardening in the matrix phase, $m_{0}=$ 0.05 and $m_{\mathrm{I}}=0.4$ in Fig. 11(b), only the solution obtained when using the residual-incremental-secant method in both phases captures the FE solution with a high accuracy.

In fact, the choice of the incremental-secant moduli to be considered corresponds to an assumption behind the proposed MFH formulation when defining the LCC. The residual-incremental-secant method should always be considered for the inclusions phase. Concerning the elasto-plastic matrix phase, the choice of keeping or not the residual stresses is governed by the relative positions of the stress and residual stress tensors with respect to the stress space origin (zero-stress state): 


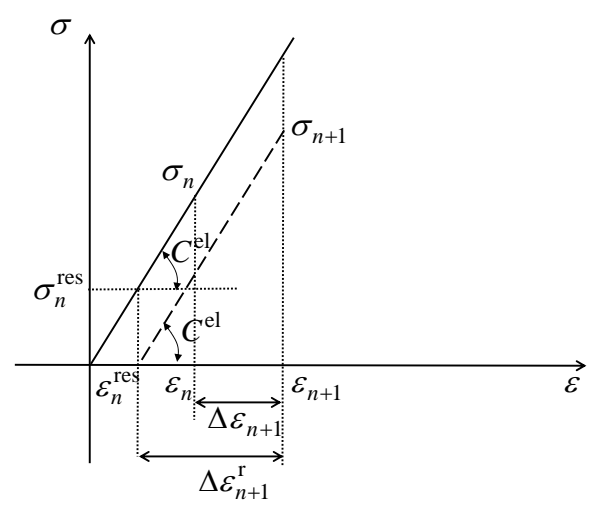

Figure 12: Configuration in an elastic matrix with the zero-incremental-secant formulation.

- The origin lies between the stress and residual stress tensors. When considering a 1D problem in tension, this means that the stress is positive and the residual stress is negative. In that case, the zeroincremental-secant operator $C^{\mathrm{S} 0}$ should be used in the matrix phase, for the same reason as for elastic inclusions. This is typically the case for composite materials made of two elasto-plastic phases for which the inclusions phase is stiffer than the matrix phase during the plastic flow, which is a common case for composite materials.

- Both stress and residual stress tensors lie on the same side with respect to the origin. When consider a $1 \mathrm{D}$ problem in tension, this means that both the stress and the residual stress are positive. In that case the residual-incremental-secant method should be considered for the matrix phase as using the zero-incremental-secant method would lead to softer prediction. Indeed as illustrated in Fig. 12 for the limiting case of a pure elastic response, the reloading path does not follow the unloading one if the residual stress is omitted, resulting in a lower stress value. This is typically a configuration of compliant elasto-plastic inclusions combined with a stiffer matrix response during the plastic flow.

We will now assert this analysis on more examples.

MMCs with low inclusions phase hardening. In the first additional example, elastic-perfectly-plastic behaviors for the inclusions and matrix phases are 


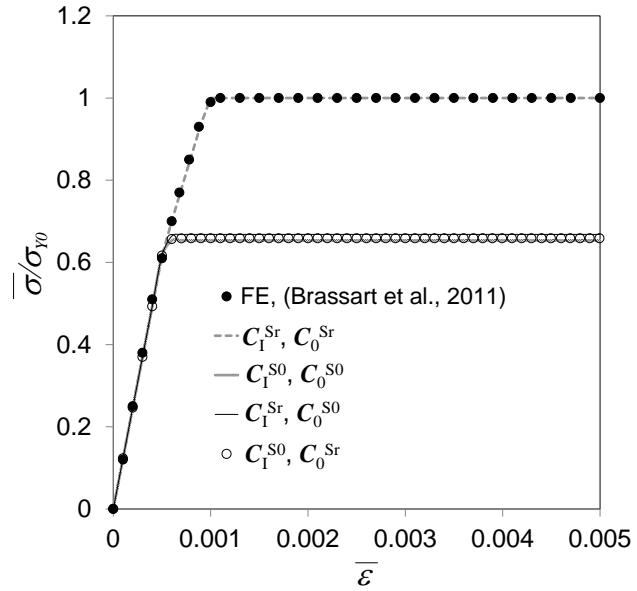

(a) Effect of the incremental-secant operators.

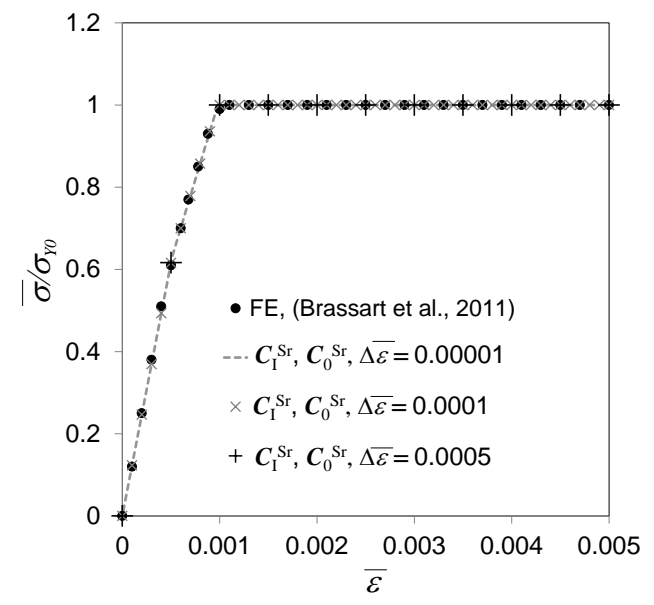

(b) Effect of the strain increment size

Figure 13: Results for composites with elastic-perfectly-plastic inclusions with a low hardening embedded in an elasto-plastic matrix. (a) Effect of the incremental-secant operators, $\Delta \bar{\varepsilon}=0.00001$. (b) Residual-incremental-secant operators, effect of the strain increment size $\Delta \bar{\varepsilon}$.

considered $\left(R_{\mathrm{I}}(p)=R_{0}(p)=0\right)$. The other material properties are the same as for the previous example

- Inclusions: $E_{\mathrm{I}}=400 \mathrm{GPa}, \nu_{\mathrm{I}}=0.2, \sigma_{Y \mathrm{I}}=75 \mathrm{MPa}$;

- Matrix: $E_{0}=75 \mathrm{GPa}, \nu_{0}=0.3, \sigma_{Y 0}=75 \mathrm{MPa}$.

Inclusions are spherical with a volume fraction of $v_{\mathrm{I}}=15 \%$. The predictions with the four combinations of the incremental-secant moduli together with FE reference results, which are found in (Brassart et al., 2011), are presented in Fig. 13(a), which shows that only the prediction obtained by using $\boldsymbol{C}^{\mathrm{Sr}}$ in both phases agrees with the FE results. Moreover, when considering the residual-incremental-secant operators, the results are found to be quasiindependent of the macro-strain increment size, see Fig. 13(b).

Another example of composite material made of two elastoplastic phases with a low hardening inclusion phase was studied by Doghri and Friebel (2005). In this case, the spherical austenite inclusions are more compliant than the ferrite matrix, see Fig. 14. The material properties of both phases, which follow the power-law hardening (53), are 


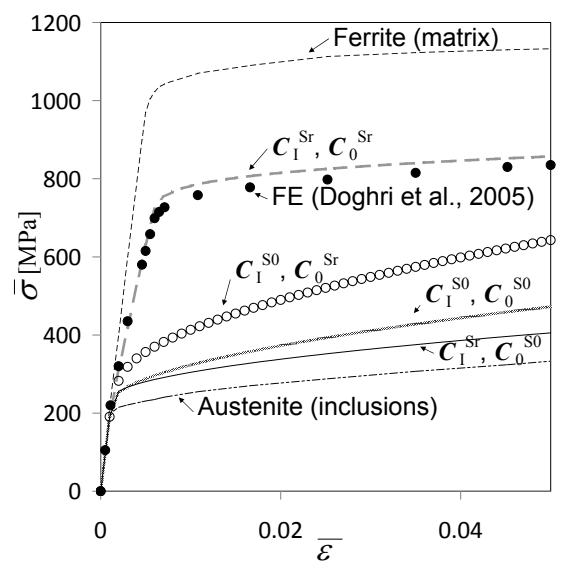

Figure 14: Results for composites with elasto-plastic inclusions with a low hardening embedded in an elasto-plastic matrix.

- Inclusions: $E_{\mathrm{I}}=179.35 \mathrm{GPa}, \nu_{\mathrm{I}}=0.3, \sigma_{Y \mathrm{I}}=202 \mathrm{MPa}, k_{\mathrm{I}}=688 \mathrm{MPa}$ and $m_{\mathrm{I}}=0.55$

- Matrix: $E_{0}=196.85 \mathrm{GPa}, \nu_{0}=0.3, \sigma_{Y 0}=600 \mathrm{MPa}, k_{0}=650 \mathrm{MPa}$ and $m_{0}=0.06$.

The inclusions volume fraction of $v_{\mathrm{I}}=35 \%$. One more time, the solution obtained by using $C^{\mathrm{Sr}}$ in both phases agrees with the FE results provided by Doghri and Friebel (2005), see Fig. 14.

From the examples above, we can say that good estimations can be obtained by using $\boldsymbol{C}_{\mathrm{I}}^{\mathrm{Sr}}$ and $\boldsymbol{C}_{0}^{\mathrm{Sr}}$ in the proposed $\mathrm{MFH}$ formulation when both phases are elasto-plastic materials and if, during the plastic flow, the inclusions response is not stiffer than the matrix phase. In other cases, such a combination is too stiff and the zero-incremental-secant operator $C_{0}^{\mathrm{S} 0}$ should be considered in the matrix phase. However as previously said, it is believed that formulating the framework by considering second-moment-statistical values will allows considering the residual stress in both phases in any cases.

\section{Conclusions}

In this work, a new incremental-secant MFH process for composites made of elasto-plastic constituents was proposed. In this approach, an unloading of the composite material is virtually performed to estimate the residual 
strains in each phase, before applying a secant approach on the strain increments, which differ in each phase. In order to define the LCC, two secant operators were defined. The first one, the residual-incremental-secant operator, is defined from the phase residual stress. The second operator, the zero-incremental-secant operator, is defined from a stress-free state in the phase.

The method was then applied on several problems and was compared to other existing MFH methods and to direct FE simulations. These examples showed that for composites with inclusions hardening law exhibiting a stiffness lower than or of the same order as the one of the matrix material, using the residual-incremental-secant operator for both phases leads to accurate predictions. However, for composites whose elasto-plastic inclusions phase is much stiffer during the plastic flow than the elasto-plastic matrix material response and for elastic inclusions embedded in an elasto-plastic matrix, the zero-incremental-secant operator should be used in the matrix phase to avoid over-stiff predictions. As discussed in the paper, the rigorous criterion is actually based on the relative positions of the stress and residual stress tensors of the matrix phase with respect to the stress space origin. In case they lie on different sides of the stress space origin, the zero-incremental-secant operator should be used in the matrix phase.

With this restriction on the choice of the matrix operator, the method has been shown to predict the macro-stress with an accuracy level similar to, or better than, the one of the first order incremental-tangent MFH method. In particular, for short glass fibers reinforced polyamide, the new incrementalsecant method has a degree of accuracy higher than the one reached with the first order incremental-tangent method. The incremental-secant method can also capture the solution under non-monotonic non-proportional loading, contrarily to the incremental-tangent approach.

The advantages of the method lie in the simple formulation and implementation. Moreover, as the LCC is defined from secant operators which are naturally isotropic, the method does not require ad-hoc isotropisation of these operators when computing the Eshelby or concentration tensors needed in the MFH.

In the near future, the method will be extended to the damaged case. When considering a composite whose matrix phase exhibits a damaging process, the inclusions phase can be unloaded during the softening stage of the matrix. The incremental-secant approach will allow this complex behavior to be captured, ensuring a more accurate prediction of the scheme as compared 
to the incremental-tangent method.

Finally, based on the good accuracy obtained by this first-moment method, the method opens perspective for its extension to a second-moment form.

\section{Appendix A. Tensorial operations and notations}

- Dots and colons are used to indicate tensor products contracted over one and two indices respectively:

$$
\begin{aligned}
\boldsymbol{u} \cdot \boldsymbol{v}=u_{i} v_{i}, & (\boldsymbol{a} \cdot \boldsymbol{u})_{i}=a_{i j} u_{j} \\
(\boldsymbol{a} \cdot \boldsymbol{b})_{i j}=a_{i k} b_{k j}, & \boldsymbol{a}: \boldsymbol{b}=a_{i j} b_{j i} \\
(\boldsymbol{C}: \boldsymbol{a})_{i j}=C_{i j k l} a_{l k}, & (\boldsymbol{C}: \boldsymbol{D})_{i j k l}=C_{i j m n} D_{n m k l} .
\end{aligned}
$$

- Dyadic products are designated by $\otimes$ :

$$
(\boldsymbol{u} \otimes \boldsymbol{v})_{i j}=u_{i} v_{j}, \quad(\boldsymbol{a} \otimes \boldsymbol{b})_{i j k l}=a_{i j} b_{k l} .
$$

- Symbols 1 and $I$ designate the second- and fourth-order symmetric identity tensors respectively:

$$
\mathbf{1}_{i j}=\delta_{i j}, \quad \boldsymbol{I}_{i j k l}=\frac{1}{2}\left(\delta_{i k} \delta_{j l}+\delta_{i l} \delta_{j k}\right),
$$

where $\delta_{i j}=1$ if $i=j, \delta_{i j}=0$ if $i \neq j$.

- The spherical and deviatoric operators are $\boldsymbol{I}^{\mathrm{vol}}$ and $\boldsymbol{I}^{\mathrm{dev}}$ respectively:

$$
\boldsymbol{I}^{\mathrm{vol}} \equiv \frac{1}{3} \mathbf{1} \otimes \mathbf{1}, \quad \boldsymbol{I}^{\mathrm{dev}}=\boldsymbol{I}-\boldsymbol{I}^{\mathrm{vol}}
$$

so that for symmetric tensors $a_{i j}=a_{j i}$ we have:

$$
\boldsymbol{I}^{\mathrm{vol}}: \boldsymbol{a}=\frac{1}{3} a_{m m} \mathbf{1}, \quad \boldsymbol{I}^{\mathrm{dev}}: \boldsymbol{a}=\boldsymbol{a}-\frac{1}{3} a_{m m} \mathbf{1}=\operatorname{dev}(\boldsymbol{a}) .
$$

$\boldsymbol{I}^{\mathrm{vol}}$ and $\boldsymbol{I}^{\mathrm{dev}}$, and can be written as follows 


\section{Appendix B. Derivation of the closed-form expressions for the incremental-secant method}

Appendix B.1. Residual-incremental-secant operator $\boldsymbol{C}^{\text {Sr }}$ from

The evaluation of $\frac{\partial C^{\mathrm{Sr}}}{\partial \varepsilon}$, which will be used in the MFH scheme, follows

$$
\frac{\partial \boldsymbol{C}^{\mathrm{Sr}}}{\partial \boldsymbol{\varepsilon}}=\frac{\partial}{\partial \Delta \varepsilon^{\mathrm{r}}}\left(3 \kappa^{\mathrm{r}} \boldsymbol{I}^{\mathrm{vol}}+2 \mu_{s}^{\mathrm{r}} \boldsymbol{I}^{\mathrm{dev}}\right): \frac{\partial \Delta \varepsilon^{\mathrm{r}}}{\partial \boldsymbol{\varepsilon}}=2 \boldsymbol{I}^{\mathrm{dev}} \otimes \frac{\partial \mu_{s}^{\mathrm{r}}}{\partial \Delta \varepsilon^{\mathrm{r}}} .
$$

Using $\frac{\partial \Delta \boldsymbol{\sigma}^{\mathrm{eq}}}{\partial \Delta \boldsymbol{\sigma}^{\mathrm{r}}}=\frac{3}{2} \frac{\Delta \boldsymbol{s}}{\Delta \boldsymbol{\sigma}^{\mathrm{eq}}}, \frac{\partial \Delta \boldsymbol{s}}{\partial \Delta \boldsymbol{\sigma}^{\mathrm{r}}}=\boldsymbol{I}^{\mathrm{dev}}, \frac{\partial \Delta \boldsymbol{\varepsilon}^{\mathrm{eq}}}{\partial \Delta \varepsilon^{\mathrm{r}}}=\frac{2}{3} \frac{\Delta \boldsymbol{e}}{\Delta \boldsymbol{\varepsilon}^{\mathrm{eq}}}$ and Eq. (27), this last relation becomes

$$
\frac{\partial \boldsymbol{C}^{\mathrm{Sr}}}{\partial \boldsymbol{\varepsilon}}=2 \boldsymbol{I}^{\mathrm{dev}} \otimes\left[\frac{1}{6 \mu_{s}^{\mathrm{r}}\left(\Delta \varepsilon^{\mathrm{eq}}\right)^{2}} \Delta \boldsymbol{s}: \boldsymbol{C}^{\mathrm{alg}}-\frac{2}{3} \mu_{s}^{\mathrm{r}} \frac{\Delta \boldsymbol{e}}{\left(\Delta \varepsilon^{\mathrm{eq}}\right)^{2}}\right],
$$

where $C^{\text {alg }}$ is the derivative of the stress increment with respect to the strain increment, which is obtained from the constitutive law of the material. Due to the modification of the return mapping algorithm, this expression is sligthly changed compared to the usual one and reads

$$
\boldsymbol{C}^{\mathrm{alg}}=\boldsymbol{C}^{\mathrm{el}}-\frac{\left(2 \mu^{\mathrm{el}}\right)^{2}}{h} \boldsymbol{N} \otimes \boldsymbol{N}-\frac{\left(2 \mu^{\mathrm{el}}\right)^{2} \Delta p}{\left(\boldsymbol{\sigma}_{n+1}^{\mathrm{tr}}-\boldsymbol{\sigma}_{n}^{\mathrm{res}}\right)^{\mathrm{eq}}}\left(\frac{3}{2} \boldsymbol{I}^{\mathrm{dev}}-\boldsymbol{N} \otimes \boldsymbol{N}\right),
$$

with $h=\frac{1}{3} \boldsymbol{N}:\left(\frac{3}{2} \frac{\left(\boldsymbol{\sigma}_{n+1}\right)^{\mathrm{dev}}}{\left(\boldsymbol{\sigma}_{n+1}\right)^{\mathrm{eq}}}\right)^{-1} \frac{\partial R}{\partial p}+3 \mu^{\mathrm{el}}$.

Appendix B.2. Zero-incremental-secant operator $\boldsymbol{C}^{S 0}$ $\frac{2}{3} \frac{\Delta e}{\Delta \varepsilon^{\mathrm{eq}}}$

The evaluation of $\frac{\partial \boldsymbol{C}^{\mathrm{S} 0}}{\partial \boldsymbol{\varepsilon}}$ is obtained using $\frac{\partial \boldsymbol{\sigma}^{\mathrm{eq}}}{\partial \boldsymbol{\sigma}}=\frac{3}{2} \frac{\boldsymbol{s}}{\boldsymbol{\sigma}^{\mathrm{eq}}}, \frac{\partial \boldsymbol{s}}{\partial \boldsymbol{\sigma}}=\boldsymbol{I}^{\mathrm{dev}}, \frac{\partial \Delta \varepsilon^{\mathrm{eq}}}{\partial \Delta \varepsilon^{\mathrm{r}}}=$

$$
3 \mu_{s}^{0}=\frac{\sigma^{\mathrm{eq}}}{\Delta \varepsilon^{\mathrm{eq}}} .
$$

and $\kappa^{0}=\kappa^{\mathrm{el}}$. This operator is readily obtained by

$$
\frac{\partial \boldsymbol{C}^{\mathrm{S} 0}}{\partial \boldsymbol{\varepsilon}}=2 \boldsymbol{I}^{\mathrm{dev}} \otimes\left[\frac{1}{6 \mu_{s}^{0}\left(\Delta \varepsilon^{\mathrm{eq}}\right)^{2}} \boldsymbol{s}: \boldsymbol{C}^{\mathrm{alg}}-\frac{2}{3} \mu_{s}^{0} \frac{\Delta \boldsymbol{e}}{\left(\Delta \varepsilon^{\mathrm{eq}}\right)^{2}}\right] .
$$

For this zero-incremental-secant approach, the direction of the normal corresponds strictly to the radial return mapping assumption, and the classical expression of $C^{\text {alg }}$ is recovered:

$$
\boldsymbol{C}^{\mathrm{alg}}=\boldsymbol{C}^{\mathrm{el}}-\frac{\left(2 \mu^{\mathrm{el}}\right)^{2}}{h_{0}} \boldsymbol{N} \otimes \boldsymbol{N}-\frac{\left(2 \mu^{\mathrm{el}}\right)^{2} \Delta p}{\left(\boldsymbol{\sigma}_{n+1}^{\mathrm{tr}}\right)^{\mathrm{eq}}}\left(\frac{3}{2} \boldsymbol{I}^{\mathrm{dev}}-\boldsymbol{N} \otimes \boldsymbol{N}\right),
$$

with $h_{0}=3 \mu^{\mathrm{el}}+\frac{\mathrm{d} R}{\mathrm{~d} p}>0$. 


\section{Appendix C. Residual stress vector}

The equation to be satisfied at the end of the MFH procedure is Eq. (35). Multiplying Eq. (33) by $\boldsymbol{B}^{\epsilon}\left(\boldsymbol{I}, \overline{\boldsymbol{C}}_{0}^{\mathrm{S}}, \overline{\boldsymbol{C}}_{\mathrm{I}}^{\mathrm{S}}\right)$ and using (35) lead to

$$
v_{0} \Delta \varepsilon_{\mathrm{I} n+1}^{\mathrm{r}}+v_{\mathrm{I}} \boldsymbol{B}^{\epsilon}\left(\boldsymbol{I}, \overline{\boldsymbol{C}}_{0}^{\mathrm{S}}, \overline{\boldsymbol{C}}_{\mathrm{I}}^{\mathrm{S}}\right): \Delta \varepsilon_{\mathrm{I} n+1}^{\mathrm{r}}=\boldsymbol{B}^{\epsilon}\left(\boldsymbol{I}, \overline{\boldsymbol{C}}_{0}^{\mathrm{S}}, \overline{\boldsymbol{C}}_{\mathrm{I}}^{\mathrm{S}}\right): \Delta \bar{\varepsilon}_{n+1}^{\mathrm{r}} .
$$

With the M-T assumption the strain concentration tensor follows from (4), and Eq. (C.1) reads

$$
\Delta \varepsilon_{\mathrm{I} n+1}^{\mathrm{r}}+v_{0} S:\left[\left(\overline{\boldsymbol{C}}_{0}^{\mathrm{S}}\right)^{-1}: \overline{\boldsymbol{C}}_{\mathrm{I}}^{\mathrm{S}}-\boldsymbol{I}\right]: \Delta \varepsilon_{\mathrm{I} n+1}^{\mathrm{r}}=\Delta \bar{\varepsilon}_{n+1}^{\mathrm{r}},
$$

or again $\boldsymbol{F}=0$ with

$$
\boldsymbol{F}=\overline{\boldsymbol{C}}_{0}^{\mathrm{S}}:\left[\Delta \varepsilon_{\mathrm{I} n+1}^{\mathrm{r}}-\frac{1}{v_{0}} S^{-1}:\left(\Delta \varepsilon_{\mathrm{I} n+1}^{\mathrm{r}}-\Delta \bar{\varepsilon}_{n+1}^{\mathrm{r}}\right)\right]-\overline{\boldsymbol{C}}_{\mathrm{I}}^{\mathrm{S}}: \Delta \varepsilon_{\mathrm{I} n+1}^{\mathrm{r}} \cdot(\mathrm{C}
$$

In order to satisfy $\boldsymbol{F}=0, \mathrm{Eq}$. (C.3) is linearized as

$$
\mathrm{d} \boldsymbol{F}=\frac{\partial \boldsymbol{F}}{\partial \varepsilon_{\mathrm{I}}}: \mathrm{d} \Delta \varepsilon_{\mathrm{I}}^{\mathrm{r}}+\frac{\partial \boldsymbol{F}}{\partial \varepsilon_{0}}: \mathrm{d} \Delta \varepsilon_{0}^{\mathrm{r}}+\frac{\partial \boldsymbol{F}}{\partial \bar{\varepsilon}}: \mathrm{d} \Delta \bar{\varepsilon}^{\mathrm{r}}
$$

When solving $\boldsymbol{F}=0$ at constant $\Delta \overline{\boldsymbol{\varepsilon}}^{\mathrm{r}}$, as $v_{0} \Delta \varepsilon_{0 n+1}^{\mathrm{r}}+v_{\mathrm{I}} \Delta \varepsilon_{\mathrm{I}_{n+1}}^{\mathrm{r}}$ is also constant, the iteration process relies on $\mathrm{d} \boldsymbol{F}=\boldsymbol{J}: \mathrm{d} \varepsilon_{\mathrm{I}}$ with

$$
\begin{aligned}
\boldsymbol{J}= & \frac{\partial \boldsymbol{F}}{\partial \varepsilon_{\mathrm{I}}}+\frac{\partial \boldsymbol{F}}{\partial \varepsilon_{0}}: \frac{\partial \boldsymbol{\varepsilon}_{0}}{\partial \varepsilon_{\mathrm{I}}} \\
= & \left.\left.\overline{\boldsymbol{C}}_{0 n+1}^{\mathrm{S}}:\left[\boldsymbol{I}-\boldsymbol{S}^{-1}\right]-\overline{\boldsymbol{C}}_{\mathrm{I} n+1}^{\mathrm{S}}-\frac{\partial \overline{\boldsymbol{C}}_{\mathrm{I} n+1}^{\mathrm{S}}: \Delta \boldsymbol{\varepsilon}_{\mathrm{I} n+1}^{\mathrm{r}}-}{\partial \boldsymbol{\varepsilon}_{\mathrm{I}}}\right]-\frac{\left(\Delta \boldsymbol{\varepsilon}_{\mathrm{I} n+1}^{\mathrm{r}}-\Delta \overline{\boldsymbol{\varepsilon}}_{n+1}^{\mathrm{r}}\right)}{v_{0}}\right]- \\
& \frac{v_{\mathrm{I}}}{v_{0}} \frac{\partial \overline{\boldsymbol{C}}_{0 n+1}^{\mathrm{S}}}{\partial \varepsilon_{0}}:\left[\Delta \varepsilon_{\mathrm{I} n+1}^{\mathrm{r}}-\boldsymbol{S}^{-1}: \frac{\partial \boldsymbol{S}}{\partial \boldsymbol{\varepsilon}_{0}}-\right. \\
& \frac{v_{\mathrm{I}}}{v_{0}^{2}} \overline{\boldsymbol{C}}_{0 n+1}^{\mathrm{S}} \otimes\left(\Delta \boldsymbol{\varepsilon}_{\mathrm{I}_{n+1}}^{\mathrm{r}}-\Delta \overline{\boldsymbol{\varepsilon}}_{n+1}^{\mathrm{r}}\right)::\left(\boldsymbol{S}^{-1} \otimes \boldsymbol{S}^{-1}\right):: \\
& \frac{v_{\mathrm{I}}}{v_{0}} \overline{\boldsymbol{C}}_{0 n+1}^{\mathrm{S}}: \boldsymbol{S}^{-1},
\end{aligned}
$$

where $\frac{\partial \overline{\boldsymbol{C}}_{\mathrm{r}}^{\mathrm{S}}}{\partial \boldsymbol{\varepsilon}_{r}}$ results from either (B.2) or (B.5). The derivative of the Eshelby tensor is reported in Appendix D. 
Once $\boldsymbol{F}=0$ is satisfied, the effect on the strain increment in each phase of a variation $\mathrm{d} \Delta \bar{\varepsilon}^{\mathrm{r}}$ can directly be obtained by constraining $\mathrm{d} \boldsymbol{F}=0$, and Eq. (C.4) leads to ${ }^{6}$

$$
0=\frac{\partial \boldsymbol{F}}{\partial \varepsilon_{\mathrm{I}}}: \mathrm{d} \Delta \varepsilon_{\mathrm{I}}^{\mathrm{r}}+\frac{\partial \boldsymbol{F}}{\partial \varepsilon_{0}}: \mathrm{d} \Delta \varepsilon_{0}^{\mathrm{r}}+\frac{\partial \boldsymbol{F}}{\partial \bar{\varepsilon}}: \mathrm{d} \Delta \bar{\varepsilon}^{\mathrm{r}}
$$

or again

$$
\frac{\partial \varepsilon_{\mathrm{I}}}{\partial \bar{\varepsilon}}=-\boldsymbol{J}^{-1}: \frac{\partial \boldsymbol{F}}{\partial \bar{\varepsilon}}
$$

As under these circumstances $\mathrm{d} \overline{\boldsymbol{\varepsilon}}^{\mathrm{r}}=v_{0} \mathrm{~d} \boldsymbol{\varepsilon}_{0}^{\mathrm{r}}+v_{\mathrm{I}} \mathrm{d} \boldsymbol{\varepsilon}_{\mathrm{I}}^{\mathrm{r}}$, this last equation is completed by

$$
\frac{\partial \varepsilon_{0}}{\partial \bar{\varepsilon}}=\frac{1}{v_{0}}\left(\boldsymbol{I}-v_{1} \frac{\partial \varepsilon_{\mathrm{I}}}{\partial \bar{\varepsilon}}\right)
$$

\section{Appendix D. Eshelby Tensor and it is derivative}

The derivative of Eshelby tensor can be written as

$$
\frac{\partial \boldsymbol{S}}{\partial \Delta \varepsilon^{\mathrm{r}}}=\frac{\partial \boldsymbol{S}}{\partial \nu} \otimes\left(\frac{\partial \nu}{\partial \kappa} \frac{\partial \kappa}{\partial \Delta \varepsilon^{\mathrm{r}}}+\frac{\partial \nu}{\partial \mu_{s}} \frac{\partial \mu_{s}}{\partial \Delta \varepsilon^{\mathrm{r}}}\right) .
$$

One directly has

$$
\frac{\partial \kappa}{\partial \Delta \varepsilon^{\mathrm{r}}}=0
$$

and therefore,

$$
\frac{\partial \boldsymbol{S}}{\partial \Delta \varepsilon^{\mathrm{r}}}=\frac{\partial \boldsymbol{S}}{\partial \nu} \otimes \frac{\partial \nu}{\partial \mu_{s}} \frac{\partial \mu_{s}}{\partial \Delta \varepsilon^{\mathrm{r}}}
$$

\section{Acknowledgment}

The research has been funded by the Walloon Region under the agreement SIMUCOMP n 1017232 (CT-EUC 2010-10-12) in the context of the ERANET +, Matera + framework.

\footnotetext{
${ }^{6}$ Note that the derivative with respect to $\Delta \varepsilon_{r}^{\mathrm{r}}$ has the same expression as the derivative with respect to $\varepsilon_{r}$
} 


\section{References}

Aboudi, J., Pindera, M.J., Arnold, S., 2003. Higher-order theory for periodic multiphase materials with inelastic phases. International Journal of Plasticity 19, $805-847$.

Benveniste, Y., 1987. A new approach to the application of Mori-Tanaka's theory in composite materials. Mechanics of Materials 6, $147-157$.

Berveiller, M., Zaoui, A., 1978. An extension of the self-consistent scheme to plastically-flowing polycrystals. Journal of the Mechanics and Physics of Solids 26, $325-344$.

Brassart, L., Doghri, I., Delannay, L., 2010. Homogenization of elasto-plastic composites coupled with a nonlinear finite element analysis of the equivalent inclusion problem. International Journal of Solids and Structures 47, $716-729$.

Brassart, L., Stainier, L., Doghri, I., Delannay, L., 2011. A variational formulation for the incremental homogenization of elasto-plastic composites. Journal of the Mechanics and Physics of Solids 59, 2455 - 2475.

Brassart, L., Stainier, L., Doghri, I., Delannay, L., 2012. Homogenization of elasto-(visco) plastic composites based on an incremental variational principle. International Journal of Plasticity 36, $86-112$.

Carrere, N., Valle, R., Bretheau, T., Chaboche, J.L., 2004. Multiscale analysis of the transverse properties of ti-based matrix composites reinforced by sic fibres: from the grain scale to the macroscopic scale. International Journal of Plasticity 20, $783-810$.

Chaboche, J., Kanouté, P., Roos, A., 2005. On the capabilities of mean-field approaches for the description of plasticity in metal matrix composites. International Journal of Plasticity 21, 1409 - 1434.

Christman, T., Needleman, A., Nutt, S., Suresh, S., 1989a. On microstructural evolution and micromechanical modelling of deformation of a whisker-reinforced metal-matrix composite. Materials Science and Engineering: A 107, 49 -61. Poceedings of the Symposium on Interfacial Phenomena in Composites: Processing Characterization and Mechanical Properties. 
Christman, T., Needleman, A., Suresh, S., 1989b. An experimental and numerical study of deformation in metal-ceramic composites. Acta Metallurgica 37, $3029-3050$.

Doghri, I., Adam, L., Bilger, N., 2010. Mean-field homogenization of elastoviscoplastic composites based on a general incrementally affine linearization method. International Journal of Plasticity 26, 219 - 238.

Doghri, I., Brassart, L., Adam, L., Gérard, J.S., 2011. A second-moment incremental formulation for the mean-field homogenization of elasto-plastic composites. International Journal of Plasticity 27, 352 - 371.

Doghri, I., Friebel, C., 2005. Effective elasto-plastic properties of inclusionreinforced composites. study of shape, orientation and cyclic response. Mechanics of Materials 37, $45-68$.

Doghri, I., Ouaar, A., 2003. Homogenization of two-phase elasto-plastic composite materials and structures: Study of tangent operators, cyclic plasticity and numerical algorithms. International Journal of Solids and Structures 40, $1681-1712$.

Doghri, I., Tinel, L., 2005. Micromechanical modeling and computation of elasto-plastic materials reinforced with distributed-orientation fibers. International Journal of Plasticity 21, 1919 - 1940.

Dvorak, G.J., 1992. Transformation field analysis of inelastic composite materials. Proceedings: Mathematical and Physical Sciences 437, 311-327.

Dvorak, G.J., Bahei-El-Din, Y.A., Wafa, A.M., 1994. Implementation of the transformation field analysis for inelastic composite materials. Computational Mechanics 14, 201-228.

Engelen, R.A.B, M., Baaijens, F., 2003. Nonlocal implicit gradient-enhanced elasto-plasticity for the modelling of softening behaviour. International Journal of Plasticity 19, $403-433$.

Eshelby, J.D., 1957. The determination of the elastic field of an ellipsoidal inclusion, and related problems. Proceedings of the Royal Society of London. Series A, Mathematical and Physical Sciences 241, pp. 376-396. 
Geers, M., 1997. Experimental Analysis and Computational Modelling of Damage and Fracture. Ph.D. thesis. University of Technology, Eindhoven (Netherlands).

Geers, M., Kouznetsova, V., Brekelmans, A., 2010. Multi-scale computational homogenization: Trends and challenges. Journal of Computational and Applied Mathematics 234, 2175 - 2182.

Hill, R., 1965a. Continuum micro-mechanics of elastoplastic polycrystals. Journal of the Mechanics and Physics of Solids 13, 89 - 101.

Hill, R., 1965b. A self-consistent mechanics of composite materials. Journal of the Mechanics and Physics of Solids 13, $213-222$.

Jansson, S., 1992. Homogenized nonlinear constitutive properties and local stress concentrations for composites with periodic internal structure. International Journal of Solids and Structures 29, $2181-2200$.

Ji, B., Wang, T., 2003. Plastic constitutive behavior of short-fiber/particle reinforced composites. International Journal of Plasticity 19, $565-581$.

Kanouté, P., Boso, D., Chaboche, J., Schrefler, B., 2009. Multiscale methods for composites: A review. Archives of Computational Methods in Engineering 16, 31-75. 10.1007/s11831-008-9028-8.

Kouznetsova, V., Geers, M., Brekelmans, W., 2004. Multi-scale second-order computational homogenization of multi-phase materials: a nested finite element solution strategy. Computer Methods in Applied Mechanics and Engineering 193, 5525 - 5550. Advances in Computational Plasticity.

Kouznetsova, V., Geers, M.G.D., Brekelmans, W.A.M., 2002. Multiscale constitutive modelling of heterogeneous materials with a gradientenhanced computational homogenization scheme. International Journal for Numerical Methods in Engineering 54, 1235-1260.

Kröner, E., 1958. Berechnung der elastischen konstanten des vielkristalls aus den konstanten des einkristalls. Zeitschrift für Physik A Hadrons and Nuclei 151, 504-518. 10.1007/BF01337948.

Lahellec, N., Ponte Castañeda, P., Suquet, P., 2011. Variational estimates for the effective response and field statistics in thermoelastic composites 
with intra-phase property fluctuations. Proceedings of the Royal Society A: Mathematical, Physical and Engineering Science 467, 2224-2246.

Lahellec, N., Suquet, P., 2007a. On the effective behavior of nonlinear inelastic composites: I. incremental variational principles. Journal of the Mechanics and Physics of Solids 55, 1932 - 1963.

Lahellec, N., Suquet, P., 2007b. On the effective behavior of nonlinear inelastic composites: II. a second-order procedure. Journal of the Mechanics and Physics of Solids 55, $1964-1992$.

Lahellec, N., Suquet, P., 2013. Effective response and field statistics in elastoplastic and elasto-viscoplastic composites under radial and non-radial loadings. International Journal of Plasticity .

Li, G., Ponte Castañeda, P., 1994. Variational Estimates for the Elastoplastic Response of Particle-Reinforced Metal-Matrix Composites. Applied Mechanics Reviews 47, 77.

Lissenden, C., Arnold, S., 1997. Theoretical and experimental considerations in representing macroscale flow/damage surfaces for metal matrix composites. International Journal of Plasticity 13, 327 - 358 .

Masson, R., Bornert, M., Suquet, P., Zaoui, A., 2000. An affine formulation for the prediction of the effective properties of nonlinear composites and polycrystals. Journal of the Mechanics and Physics of Solids 48, $1203-$ 1227.

Mercier, S., Molinari, A., 2009. Homogenization of elasticviscoplastic heterogeneous materials: Self-consistent and Mori-Tanaka schemes. International Journal of Plasticity 25, $1024-1048$.

Molinari, A., Canova, G., Ahzi, S., 1987. A self consistent approach of the large deformation polycrystal viscoplasticity. Acta Metallurgica 35, 29832994.

Molinari, A., El Houdaigui, F., Tóth, L., 2004. Validation of the tangent formulation for the solution of the non-linear eshelby inclusion problem. International Journal of Plasticity 20, 291 - 307. 
Mori, T., Tanaka, K., 1973. Average stress in matrix and average elastic energy of materials with misfitting inclusions. Acta Metallurgica 21, 571574. Cited By (since 1996) 1814.

Moulinec, H., Suquet, P., 2003. Intraphase strain heterogeneity in nonlinear composites: a computational approach. European Journal of Mechanics A/Solids 22, $751-770$.

Peerlings, R., de Borst, R., Brekelmans, W., Ayyapureddi, S., 1996. Gradient-enhanced damage for quasi-brittle materials. Int. J. Numer. Meth. Engng 39, 3391-3403.

Peerlings, R., de Borst, R., Brekelmans, W., Geers, M., 1998. Gradientenhanced damage modelling of concrete fracture. Mech. CohesiveFrictional Mat. 3, 323-342.

Peerlings, R., Geers, M., de Borst, R., Brekelmans, W., 2001. A critical comparison of nonlocal and gradient-enhanced softening continua. Int. J. Solids Structures 38, 7723-7746.

Pettermann, H.E., Plankensteiner, A.F., Böhm, H.J., Rammerstorfer, F.G., 1999. A thermo-elasto-plastic constitutive law for inhomogeneous materials based on an incremental Mori-Tanaka approach. Computers \& Structures $71,197-214$.

Pierard, O., Doghri, I., 2006a. An enhanced affine formulation and the corresponding numerical algorithms for the mean-field homogenization of elastoviscoplastic composites. International Journal of Plasticity 22, 131 - 157.

Pierard, O., Doghri, I., 2006b. Study of various estimates of the macroscopic tangent operator in the incremental homogenization of elastoplastic composites. International Journal for Multiscale Computational Engineering $4,521-543$.

Pierard, O., Gonzlez, C., Segurado, J., LLorca, J., Doghri, I., 2007a. Micromechanics of elasto-plastic materials reinforced with ellipsoidal inclusions. International Journal of Solids and Structures 44, 6945 - 6962.

Pierard, O., LLorca, J., Segurado, J., Doghri, I., 2007b. Micromechanics of particle-reinforced elasto-viscoplastic composites: Finite element simulations versus affine homogenization. International Journal of Plasticity 23, $1041-1060$. 
Ponte Castañeda, P., 1991. The effective mechanical properties of nonlinear isotropic composites. Journal of the Mechanics and Physics of Solids 39, $45-71$.

Ponte Castañeda, P., 1992. A new variational principle and its application to nonlinear heterogeneous systems. SIAM Journal on Applied Mathematics $52,1321-1341$.

Ponte Castañeda, P., 1996. Exact second-order estimates for the effective mechanical properties of nonlinear composite materials. Journal of the Mechanics and Physics of Solids 44, $827-862$.

Ponte Castañeda, P., 2002a. Second-order homogenization estimates for nonlinear composites incorporating field fluctuations: I - theory. Journal of the Mechanics and Physics of Solids 50, $737-757$.

Ponte Castañeda, P., 2002b. Second-order homogenization estimates for nonlinear composites incorporating field fluctuations: II - applications. Journal of the Mechanics and Physics of Solids 50, $759-782$.

Segurado, J., Llorca, J., 2002. A numerical approximation to the elastic properties of sphere-reinforced composites. Journal of the Mechanics and Physics of Solids 50, $2107-2121$.

Segurado, J., Llorca, J., González, C., 2002. On the accuracy of meanfield approaches to simulate the plastic deformation of composites. Scripta Materialia 46, $525-529$.

Suquet, P., 1995. Overall properties of nonlinear composites: A modified secant moduli theory and its link with ponte castañeda's nonlinear variational procedure. Comptes Rendus de l" Académie des Sciences 320, 563-571.

Talbot, D., Willis, J., 1992. Some simple explicit bounds for the overall behaviour of nonlinear composites. International Journal of Solids and Structures 29, $1981-1987$.

Talbot, D.R.S., Willis, J.R., 1985. Variational principles for inhomogeneous non-linear media. IMA Journal of Applied Mathematics 35, 39-54. 
Talbot, D.R.S., Willis, J.R., 1987. Bounds and self-consistent estimates for the overall properties of nonlinear composites. IMA Journal of Applied Mathematics 39, 215-240.

Wieckowski, Z., 2000. Dual finite element methods in homogenization for elasticplastic fibrous composite material. International Journal of Plasticity $16,199-221$.

Wu, L., Noels, L., Adam, L., Doghri, I., 2012. Multiscale mean-field homogenization method for fiber-reinforced composites with gradient-enhanced damage model. Computer Methods in Applied Mechanics and Engineering 233-236, 164-179.

Zaoui, A., Masson, R., 2002. Modelling stress-dependent transformation strains of heterogeneous materials, in: Bahei-El-Din, Y.A., Dvorak, G.J., Gladwell, G.M.L. (Eds.), IUTAM Symposium on Transformation Problems in Composite and Active Materials. Springer Netherlands. volume 60 of Solid Mechanics and Its Applications, pp. 3-15. 